San Jose State University

SJSU ScholarWorks

Master's Theses

Master's Theses and Graduate Research

1995

\title{
A comparison of the effectiveness of the Harvard Calculus series with the traditionally taught calculus series
}

Gregory H. Allen

San Jose State University

Follow this and additional works at: https://scholarworks.sjsu.edu/etd_theses

\section{Recommended Citation}

Allen, Gregory H., "A comparison of the effectiveness of the Harvard Calculus series with the traditionally taught calculus series" (1995). Master's Theses. 969.

DOI: https://doi.org/10.31979/etd.954p-xfgd

https://scholarworks.sjsu.edu/etd_theses/969

This Thesis is brought to you for free and open access by the Master's Theses and Graduate Research at SJSU ScholarWorks. It has been accepted for inclusion in Master's Theses by an authorized administrator of SJSU ScholarWorks. For more information, please contact scholarworks@sjsu.edu. 


\section{INFORMATION TO USERS}

This manuscript has been reproduced from the microfilm master. UMI films the text directly from the original or copy submitted. Thus, some thesis and dissertation copies are in typewriter face, while others may be from any type of computer printer.

The quality of this reproduction is dependent upon the quality of the copy submitted. Broken or indistinct print, colored or poor quality illustrations and photographs, print bleedthrough, substandard margins, and improper alignment can adversely affect reproduction.

In the unlikely event that the author did not send UMI a complete manuscript and there are missing pages, these will be noted. Also, if unauthorized copyright material had to be removed, a note will indicate the deletion.

Oversize materials (e.g., maps, drawings, charts) are reproduced by sectioning the original, beginning at the upper left-hand corner and contiming from left to right in equal sections with small overlaps. Each original is also photographed in one exposure and is included in reduced form at the back of the book.

Photographs included in the original manuscript have been reproduced xerographically in this copy. Higher quality $6^{\prime \prime} \times 9^{n}$ black and white photographic prints are available for any photographs or illustrations appearing in this copy for an additional charge. Contact UMI directly to order.

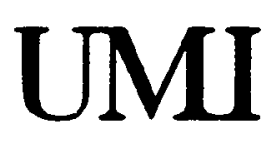

A Bell \& Howell Information Company 300 North Zeeb Road. Ann Arbor. MI 48106-1346 USA $313: 761-4700 \quad 800: 521-0600$ 



\title{
A COMPARISON \\ OF THE EFFECTIVENESS OF THE \\ HARVARD CALCULUS SERIES WITH THE \\ TRADITIONALLY TAUGHT CALCULUS SERIES
}

\author{
A Project \\ Presented to \\ The Faculty of the Department of Mathematics and Computer Science \\ San Jose State University
}

In Partial Fulfillment

of the Requirements for the Degree

Master of Arts

by

Greg Allen

May 1995 
UMI Number: 1374560

UMI Microform 1374560

Copyright 1995, by UMI Company. All rights reserved.

This microform edition is protected against unauthorized copying under Title 17, United States Code.

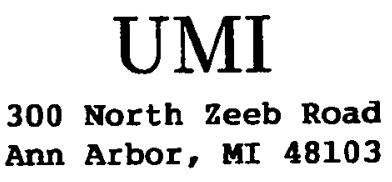


APPROVED FOR THE DEPARTMENT OF MATHEMATICS AND COMPUTER SCIENCE
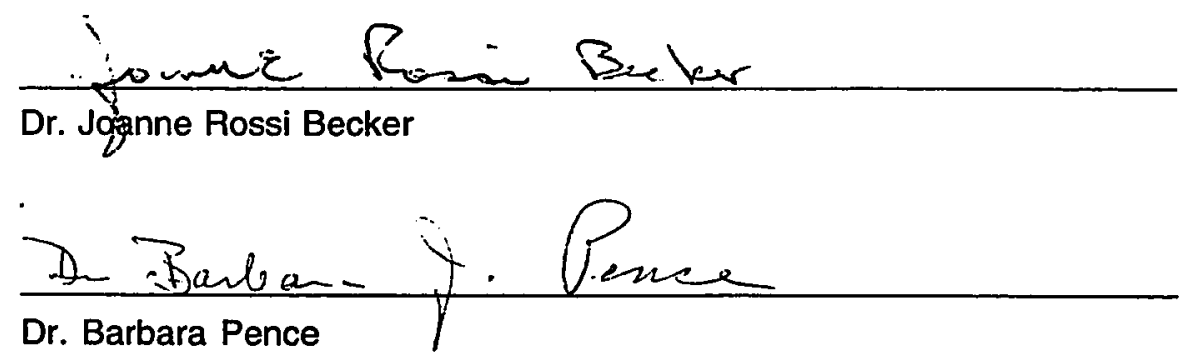

$\frac{\text { Mana \& Cayes - Hajji }}{\text { Dr. Maria Cayco-Gajic }}$

APPROVED FOR THE UNIVERSITY

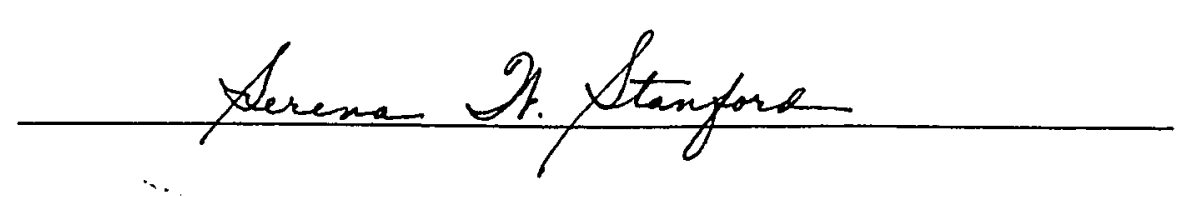


(C) 1995

Greg Allen

ALL RIGHTS RESERVED 


\section{ABSTRACT \\ ACOMPARISON \\ OF THE EFFECTIVENESS OF THE \\ HARVARD CALCULUS SERIES WITH THE \\ TRADITIONALLY TAUGHT CALCULUS SERIES}

by Greg Allen

This study compared six Harvard Calculus students with six students from traditionally taught calculus, all of whom had completed a year of calculus during the 1993-1994 school year. The students were interviewed and tested on their feelings about the course and text as well as their understanding of some of the key ideas from the first year of calculus.

The responses from the two groups indicate that the Harvard Group has a better attitude about mathematics, and is better able to work with topics across multiple representations (graphic, numeric, symbolic). Analysis of the interviews and tests also reveals that the students from the Harvard Group have a better understanding of most of the concepts examined in this study. Specifically, the Harvard Group consistently performed better than the traditional group on questions regarding continuity, differentiation, and integration. The two groups performed equally well on functions. Limits was the only area in which the traditional group performed better, although neither group did very well on this topic.

The results from this study clearly indicate that Harvard Calculus is a worthy alternative to the traditionally taught calculus. It is recommended that other Mathematics Departments offer a reform-style calculus, and perhaps follow up with a comparative study similar to this one. 


\section{ACKNOWLEDGEMENT}

To Dr. Joanne Becker, thank you for your patience and guidance. They -- along with your great editing skills -- were greatly appreciated.

To my wife Carolann, thank you for your patience (even if it did wear thin at times) and support. If you ever hear me say the three letters $P-H-D$, please take a bound copy of this thesis and hit me with it.

To my daughter Ashley, thank you for your distractions -- they may not have always been appreciated at the time, but they were very necessary. 


\section{TABLEOF CONTENTS}

Chapter 1 Introduction................................................................ page 1

Chapter 2 Review of the Literature.............................................. page 3

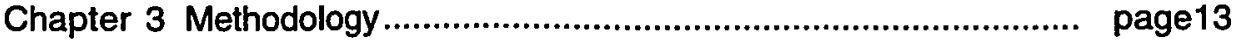

Chapter 4 Results........................................................................... page 29

Chapter 5 Conclusions................................................................. page 79

References ................................................................................. page 91 


\section{LIST OF TABLES}

Table 1 Student Assessments.......................................................... page 44

Table 2 Functions ..................................................................... page 51

Table 3 Limits ...................................................................... page 57

Table 4 Continuity ....................................................................... page 61

Table 5 Differentiation.............................................................. page 73

Table 6 Integration................................................................ page 78 


\section{CHAPTER 1}

\section{INTRODUCTION}

In their article "Cognitive Obstacles to the Learning of Calculus," Norman and Prichard claim that "the learning of calculus is in an abysmal state" (1994, p. 65). There is much evidence to support their claim. In fact, more and more evidence keeps pointing to the simple fact that although students are quite capable of solving routine, algorithmic-type calculus problems, they are very weak when it comes to understanding the key concepts of calculus, or in applying the skills they have learned to non-routine applications. And one should not be too quick to place the blame on the student. After all, if a student can successfully complete the calculus series by simply "plugging in numbers," then who is to blame? The instructor? The department? The text? Or perhaps even the curriculum? Whatever the cause, clearly a reformation of the teaching of calculus is needed.

The idea of reforming calculus is not a new one. For many years educators have been rallying for an across-the-board reformation of the calculus curriculum. In 1986, the Mathematical Association of America (MAA) published a collection of articles that emphasized the need for reform. In this publication, titled Toward a Lean and Lively Calculus, the MAA not only outlined the main problems with the current curriculum, but also suggested a new curriculum and even outlined the goals that students should meet upon completion of the calculus series. Some of the problems with current teaching practices, according to the MAA, are the following:

1) Current practice is perceived to drive students away from scientific and mathematical careers.

2) Most current courses are superficial. . . "mimicry calculus" courses [that] do not develop understanding. They fail to prepare students for applications of mathematics to their disciplines, [or to even] convey a sense of mathematics and mathematical thinking.

3) Calculus as presently taught is essentially irrelevant for the nearly $50 \%$ of the college students who do not go on to use mathematical tools in their careers. These students. . . are ill-served by current versions of the course. (p. xv)

Although that publication came out in 1986, many of the same complaints are heard today. In that same publication, the MAA suggested a "leaner" and "livelier" 
curriculum and offered some suggestions for teaching calculus that would address the above problems. Some of those suggestions are below:

1) Use complex problems from the "real world" to serve as a context for doing mathematics.

2) Replace many "show that" problems with "is it true; provide an example or counterexample" problems.

3) Assign multi-step problems that go beyond the "plug into the technique we just studied" mode.

4 ) Use graphical or tabular representations of functions as well as algebraic ones. (p. xvii).

In general, the MAA was suggesting a de-emphasis of procedures in favor of emphasizing the concepts. In order for the focus to remain on concepts and not calculations, the use of technology, such as calculators and computer algebra systems, has also been suggested.

The Harvard Calculus (Calculus Consortium at Harvard, 1994) is one of several curricula designed to address the problems the MAA outlined as well as meet the above goals. Using what is called the rule of three -- presenting topics algebraically, numerically, and geometrically -- Harvard Calculus hopes to capture the attention and imagination of the students, put an end to "mimicry calculus," and generally make calculus more relevant to the students by being more of an application-driven course. In Harvard Calculus, conceptual understanding and procedural prowess come through the exploration and investigation of real world problems.

Some immediate questions come to mind when one first hears of this "new" calculus. Does it indeed capture the imagination of the students? Do students leave the Harvard Calculus course with a better understanding of the concepts than the students who took the traditional course? Are the Harvard students able to solve procedural questions as well as the traditional students? Is there a price to pay for de-emphasizing skills in favor of concepts? This study attempts to answer some of these questions, as well as offer suggestions for future research. Of course, before one can get answers, one must know the right questions to ask, and which questions have already been asked. $A$ review of the literature pertaining to the learning of calculus is an appropriate place to start. 


\section{CHAPTER 2}

\section{REVIEW OF THE LITERATURE}

There have been a multitude of studies that conclusively illustrate that students have major difficulties when it comes to learning and understanding calculus. The limit concept seems particularly difficult to grasp, but the other main topics of calculus, such as derivatives, integrals, continuity, and even functions, are not without their difficulties. Some researchers have also shown that even students who apparently understand the key ideas and have successfully completed a course in calculus fare poorly when attempting to apply their skills to non-routine problems. Still others have shown how some students do well on procedural problems but do not have an accurate understanding of the concepts.

Of course, some of these difficulties will be encountered regardless of how the material is taught, since calculus is more complex than the courses that come before it. It is not the purpose of this paper to suggest that Havard Calculus eliminates all of these "cognitive obstacles." After all, the confusing, everyday language used to describe limits, detailed below, will most likely be encountered regardless of curriculum or pedagogy, and surely other calculus topics have their own built-in problems. However, these observed difficulties are partially responsible for the search for a better way of teaching calculus. The following sections review some of the research that has motivated calculus reform in general and Harvard Calculus in particular.

\section{DIFFICULTIES WITH THE CONCEPT OF FUNCTION}

The research available on the learning and understanding of functions is plentiful and consistent. There is virtually no argument among researchers that students have a poor understanding of what a function is and how a function can look graphically. The two main cognitive hurdles seem to be recognizing functions graphically and being able to interpret and create graphs of functions. Also, several studies have shown that students have a strong need for functions to be expressed as formulas.

A paper by Ferrini-Mundy and Graham (1994) provides an in-depth look at students' understanding of functions. For their study, they showed a group of calculus students several graphs and asked them if they could identify which graphs could represent a function. They found, as have others (Dreyfus \& Eisenberg 1982; Vinner 
1987), that students are more likely to identify a graph as a function if it is familiar and if it is continuous. The researchers detail one fairly typical research subject, whom they call Sandy. 'When Sandy classifies a graph as 'familiar' she automatically classifies this graph as a representation of a function" ( $p$. 35) regardless of whether or not the graph truly does represent a function. Her need for continuity, although inconsistent, is seen when she is shown a graph of dots in a horizontal line and she answers that the graph does not represent a function because it is just "separate dots" (p. 36). Graphs of piecewise functions also posed a problem for the research participants, since students typically think of functions as having "one equation."

In addition to not being able to recognize graphs of functions, many students are uncomfortable with graphs and are unable to interpret them. Sfard (1992) reports that "today's student often seems to regard a formula as a thing in itself, not standing for anything else. ... there is almost no connection between a graph and the underlying algebraic formula" (p. 75). Eisenberg (1993) also found that to be the case, and added that students are reluctant to use graphs for problem solving. When given a problem in which a graph would definitely clarify the solution many students either avoid or never think to sketch a diagram; those who do make a sketch often do so incorrectly. When over 300 students were asked to find the equations of the tangent lines to the circle $x^{2}+y^{2}=10$ which pass through the point $(5,5)$, Eisenberg reported that 80 percent of them did not draw a sketch of the problem.

Since students are so weak at working with and creating graphs, it should come as no surprise that they generally prefer functions to be expressed algebraically. Ferrini-Mundy and Graham (1994) describe that even when a problem is initially posed graphically, some students will first find an algebraic representation of the graph to answer any questions. Although this is not necessarily wrong, many times the underlying ideas will be lost in the notation. Perhaps if more time were spent on alternate representations of functions, such as graphs and tables, students would learn to be more flexible in their thinking and not be so attached to the formulaic approach.

\section{PROBLEMS WITH LEARNING LIMITS}

The idea of limits can be confusing for first semester calculus students, partly because in describing limits, educators often use everyday words but not necessarily with an everyday meaning. Monaghan (1991) reports that the use of words like tends to, approaches, limit and converges is likely to distract students since those words 
have everyday meanings that are not as precise as their mathematical meanings. Cornu (1991) had similar findings, stating that "in the case of the limit concept. . . the words 'tends to' and 'limit' have a significance for the students before the lessons begin" ( $p$. 154). He adds that in !ight of these preconceived definitions, "it easily happens that contradictory ideas [about limits] may be held simultaneously in the mind of an individual" (p. 155). In their report, Tall and Schwarzenberger (1978) tell how the use of the word "close" when informally describing a limit, such as "we can make the sequence] as close to [the limit] as we please," can cause confusion. "Close means near but not coincident with. . . . The informal idea of a limit may carry the hidden implication that [the sequence] can be close, but not equal to [the limit]" (p. 44). As stated above, this is one aspect of learning limits that is universal; traditionally taught calculus courses as well as the Harvard Calculus courses may experience this problem. However, if teachers are aware of the problem initially, they will be better prepared to deal with the confusing language.

Language is not the only problem with the limit concept. Studies have shown that few calculus students thoroughly understand the idea of limits (see Davis \& Vinner, 1986; Tall, 1980; Tall \& Vinner, 1981). In 1991, Williams published a siudy that confirmed what others before had found -- that students' views on limits are superficial and inconsistent. What Williams attempted to do was first find exactly what students believed a limit to be, and then create cognitive conflict by asiking unusual questions that would reveal the problems with their beliefs. What he found was that even when their view of limits was shaken, students generally viewed those unusual problems as exceptions to the rule and clung to their beliefs. Williams found that students generally think of a limit as a dynamic process, such as the process of the function moving closer and closer to the limit, or the act of substituting a finite number of elements in the domain to determine the limit at a point. He concluded that the current curriculum does not motivate students to learn the formal definition.

\section{RESEARCH ON CONTINUITY}

The research on students' views of continuity is not nearly as plentiful as the other areas looked at in this report. What research is available generally points to the strong connection students make between functions and continuity. Wilson (1994) investigated the evolving views held about functions by a preservice secondary mathematics teacher over the course of a semester. The teacher initially felt continuity was 
necessary for a graph to represent a function. She repeatedly classified graphs of functions as nonfunctions if the graphs "looked funny" or were "disjoint or not continuous" (p. 354). At one point, referring to a discontinuous graph, she commented "I'm thinking that a function can be graphed so that it's continuous" (p. 354).

Ferrini-Mundy and Graham's (1994) research subject Sandy had similar misconceptions of functions and continuity. As reported in the above section on functions, she was much more likely to classify a graph as a function if the graph was continuous. However, the representation of the function played a key role in Sandy's determining whether or not the function was continuous. If the function was presented graphically, she judged it to be continuous as long as it had no jumps or breaks. If the function was presented algebraically, she judged it to be continuous as long as the function was defined for all real numbers. 'She does seem to equate a 'jump' in the graph with a point at which the function is undefined" (p. 39). Sandy was quite puzzled when she encountered graphs of discontinuous functions that are defined everywhere.

\section{STUDENTS' UNDERSTANDING OF THE DERIVATIVE}

Students' understanding of the derivative is yet another area of concern for educators. Although it does not have the same "cognitive obstacles" as the limit concept, current methods of teaching calculus seem to leave the student with a very primitive idea of what a derivative is. In fact, most students' understanding of the derivative is essentially a set of rules for differentiating functions.

In 1983, Orton (1983b) conducted a large scale qualitative study to investigate student understanding of differentiation. For his study, he tested 110 mathematics majors with a wide range of ability. Orton found that the students did weil when asked about routine aspects of differentiation, but the students fared poorly on conceptual questions, and showed little intuitive understanding of the derivative. He concluded:

An obvious educational implication of the study is that the foundations of calculus need to be returned to and developed anew at various times throughout the students' mathematical education. A first approach to differentiation may be based largely on numerical and graphical explorations assisted by an electronic calculator. ... the rule for differentiating is not then given as a rule to be learned, it is discovered as a summary of investigatory work carried out by students. (p. 244) 
Ferrini-Mundy and Graham (1994) also found this "primitive" understanding of differentiation. They document one student who had no problem computing derivatives using algorithms but had no idea what the tangent line had to do with the derivative of the function. Additionally, Heid (1988) reported this gap between procedural prowess and conceptual understanding:

Students successfully complete the traditional course unable to approximate derivative values for functions whose derivative rules they do not know. Even the notion of derivative as slope or rate of change, or of second derivative as a measure of concavity, fades quickly with disuse because students learn to rely on memorized procedures for a small number of exercise types. (p. 9)

Scher (1993) found that students had the hardest time with differentiation problems when the question was presented in non-algebraic form. He found that students showed very little insight about the derivative when the student was working with a graph or table. Even questions that asked in which part of the graph would the derivative have the greatest value gave many of the research subjects difficulty. Scher concluded that "maybe it is time that we commit to calculus curricula that regard algebra, graphs, and tables as equally deserving of our teaching time" (p. 17).

\section{RESEARCH ON INTEGRATION}

Orton (1983a) also surveyed students on their understanding of integration (it was actually part of the study that examined students' views of differentiation). His findings were again disappointing. He found, consistent with his results on the derivative reported above, that student understanding of the integral was, for the most part, limited to basic techniques of integration. Orton found that the procedure of dissecting an area or volume, making use of the limit process, and the reasons why this process should work, were not part of most students' understanding of the integral.

Ferrini-Mundy and Graham (1994) had similar findings. They report that Sandy's "conceptions of integral were very thin and defined mainly by procedural emphases" (p. 33). They also said that Sandy, as well as other subjects, "interprets the integral as a signal to 'do something.' This is consistent with the view of function as a formula into which a value must be substituted, rather than an entity whose characteristics can be studied" (p. 41). Sandy also was confused on the constant of integration for indefinite integrals, thinking the constant represented an arbitrary 
value along the x-axis. She clearly has a poor understanding of this important area of calculus.

\section{STUDENTS' INABILITY TO TRANSFER LEARNING}

If the poor understanding of the central calculus concepts is not enough to convince one of the need for change, one should look at Selden, Selden, and Mason's 1994 report "Even Good Calculus Students Can't Solve Nonroutine Problems." They found that in addition to the students having a low-level of conceptual understanding, the students could not generalize and transfer what they had learned to problems they had not seen before. Selden, Selden, and Mason tested several students who had completed the first quarter of calculus with a grade of an A or a $B$. The test was in two parts, with a nonroutine test given initially, followed by a routine test. The routine test was mostly computational and made up of simple questions one would normally see in a first calculus course. The nonroutine test "required the students to combine what should have been familiar techniques and concepts in a way new to them, but the solutions are no more complex than sample problems given during the course" (p. 21). It should also be pointed out that $95 \%$ of the students in their survey were enrolled in the second quarter of calculus at the time of the testing, so the material should have been fairly fresh.

What Selden, Selden, and Mason found was disturbing, to say the least. Almost two-thirds of these $A$ and $B$ students could not complete a single non-routine problem, and $42 \%$ did not make substantial progress on any of the nonroutine problems. By using the routine test as an indicator, it was apparent that these results were not caused by a lack of factual knowledge. It seems that transferring the knowledge to a non-routine situation is where the problem lies. Perhaps even more disturbing was their discovery that over half of the participants used no calculus at all -- the majority of students preferred algebraic or arithmetic techniques. "They seemed to have better access to these elementary techniques, but were often unable to recognize situations in which their use is inappropriate" (p. 25). Clearly even the best students are not leaving their calculus courses with a thorough understanding of the key concepts. Commenting on this study, Dubinsky and Kaput state that "there is every reason to believe that this result applies to hundreds of thousands of students in the United States. . . . the direct message should be quite disheartening for those who believe in the current curriculum" (1994, p. 17). 


\section{SUMMARY OF THE LITERATURE}

In summary, the research has consistently shown that students' understanding of the main topics of calculus is shallow and superficial. They typically complete their calculus course with plenty of procedural knowledge but little conceptual understanding. Also, Ferrini-Mundy and Graham (1994) observed that students hold "competing, conflicting conceptions and conclusions. . quite comfortably and routinely" (p. 43). They recommend the development of a new calculus curriculum, as have several others (see Norman \& Prichard, 1994; Orton, 1983a; Scher, 1993; Selden \& Selden, 1994).

Of course, when reading these recommendations for a new curriculum, one might wonder if any alternative approaches to teaching calculus have been tried and tested for effectiveness. Although the research is not nearly as plentiful, there have been some studies conducted that do in fact show that improvements can be made -- students can leave calculus with a better grasp of the concepts than that with which they are currently leaving.

\section{ALTERNATIVE APPROACHES TO TEACHING CALCULUS}

Palmiter (1991) did a study in which she taught a 10-week integral calculus course that allowed the students to use a computer algebra system called MACSYMA. That allowed Palmiter to concentrate on concepts instead of calculations. The computer was used for computations of integrals on both homework and in-class exams. There was also a control group used in the study; this group was taught integral calculus in the traditional manner, by a different instructor. Both groups used the same texts and covered the same material. The experimental group covered the material in half the time, so the remaining five weeks were spent on pencil-and-paper techniques of integration, so as not to impede their work in future classes when MACSYMA would not be available to them. The size of each group was approximately the same (40 students in the experimental group, 41 students in the control group).

Each group was given identical computational and conceptual exams, each exam having a total of 100 possible points. Each group took the exams after the course material was covered, so the test group took the exams after the first five weeks of the course, while the traditional group took them after the 10-week course was completed. Since the experimental group was allowed to use MACSYMA on the computational exam, they were only given one hour to complete the computational exam compared to the 
traditional group's two hours. They were all given the same amount of time for the conceptual exam.

If Palmiter only discovered an improvement in computational scores, that would not be very noteworthy, since, after all, the experimental group was allowed to use computers for their computations. What she found was an improvement in computational scores and much more. On the computational exam, the experimental group averaged 90 points while the control group averaged 69.6 points (with standard deviations of 13.3 and 24.2, respectively). On the conceptual exam, the research group averaged 89.8 points with a standard deviation of 15.9 , while the traditional group averaged 72 points with a standard deviation of 21.4. Also, the experimental group did at least as well as the control group in future calculus classes. Confidence was increased in the experimental group as well, with $85 \%$ of the students in the experimental group saying they felt confident about continuing the calculus series compared with $68 \%$ of the control group.

Palmiter warns the reader against reading too much into her results, since the subjects in her study knew they were participating in a study (research has shown that subjects perform better when they are aware they are involved in a study). Additionally, the control group was tested after 10 weeks while the experimental group took the exams after five weeks, so retention may have played a role. However, one cannot disregard her findings. She has shown we can improve the status quo.

Heid (1988) has also reported improvements in concept acquisition when teaching calculus. For her study, Heid taught two sections of Applied Calculus that differed from traditionally taught Applied Calculus courses in two significant ways: first, the course stressed concept acquisition over computational skills; and secondly, it developed these concepts with a wide range of representations, such as tables, graphs, data sets, and various applications.

Like Palmiter's study, Heid's calculus class emphasized concepts by allowing the students to use a computer for calculations. There was some time spent on computation and procedural skills, but for the most part the students focused on learning the concepts and, as Heid stated, "us(ing) the meaning of the concepts to analyze problem situations" (p. 6). Critical thinking was encouraged, as was analyzing applications in other than formulaic modes. A lot of time was spent examining and analyzing tables and graphs, such as noting the relationship between the graphs of a function and its derivative, or approximating the solution to an optimization problem by studying computer generated graphs and tables. Approximations were often regarded as more important than exact 
answers. Heid felt that it was important for the class to be able to use graphical and numerical representations, since she had found that typical calculus courses rarely strayed from algebraic or formulaic representations.

Twenty students were subsequently interviewed by Heid: fifteen from the experimental class, and five from a comparison class. Heid's interviews were on concepts only, and she too found that concept acquisition improved:

On all but 2 of the 16 parts of the conceptual questions, both experimental sections outscored the comparison section. For only one question did the comparison section outscore both experimental sections. The students in the experimental classes were better able to draw conclusions about slopes, identify portions of graphs reflecting given quantitative statements, translate mathematical statements into conclusions about an applied situation, interpret facts about derivatives, explain a theoretical basis for a derivative rule, use formulas to identify which of two populations is growing more rapidly, and match exponential and logarithmic functions with their graphs. Their superior performance on these items required, in each case, the translation of a mathematical idea in one form to an equivalent representation in another form. The students in the comparison class were better able to match the graph of a function, given its derivative. (p. 18)

Perhaps even more important was the apparent depth of understanding the experimental group had. During Heid's interviews, she noticed that the experimental group generally displayed a greater conceptual understanding and "a broader array of appropriate associations" (p. 15). There were also occasions when students from the experimental group would reason from first principles; that was not the case for the control group. It should also be noted that for the class final, which was the same for all classes and was a skills test, the experimental group did almost as well as the group that had practiced skills all semester. In fact, the difference was negligible -- the experimental group averaged 110 out of 200 and the comparison class averaged 117 out of 200 .

Like Palmiter, Heid also cautions against the over-interpretation of her results. The size of the groups she interviewed was not uniform, and for the final examination, the comparison class had 100 students while the experimental group had only 35 . Also, since she taught the experimental group, the depth of their responses may have been prompted by the rapport they felt with her, although Heid took steps to become farniliar with the participants from the control group. Nevertheless, her results should speak for themselves. De-emphasizing procedural skills in the calculus curriculum evidently 
does little or no harm, and yet allows for a more thorough examination and understanding of the central concepts. The evidence strongly suggests that students benefit with an increased level of understanding.

\section{CONCLUSION}

There is no question that the way we teach calculus, and maybe even mathematics in general, is changing. The MAA (1995) estimates that $32 \%$ of all students who took a calculus course in the Spring of 1994 were in a reform course. Of course, reform courses differ from campus to campus, but they all had the element of de-emphasizing procedures and stressing concepts. Some courses also used technology, such as graphing calculators or a computer algebra system, or both. Other elements of reform courses include open-ended projects, in-class oral presentations and writing assignments.

This is not to imply that every educator agrees that reform is necessary. Indeed, there is probably not a single mathematics department in the country that has virtually every faculty member in agreement over the topic of calculus reform. Even those who agree that reform is necessary do not always agree on what changes should be made. However, it seems to this writer that to simply ignore the research detailed in this chapter and not do anything to try to improve the learning of calculus would be doing the students a disservice.

The MAA has suggested a more application-driven course, de-emphasizing technique in favor of concepts, with more open-ended questions and a greater mix of representations. Technology has also been suggested as a way to help accomplish these goals. Harvard Calculus is an application-driven course, and addresses the above concerns. Harvard Calculus was designed with the hopes to improve the current situation. Whether or not it lives up to its promise will be examined in Chapter 4. 


\section{CHAPTER 3}

\section{METHODOLOGY}

This study was conducted by interviewing and testing a group of students from a large western university. With an enrollment of approximately 26,000 students, the university offers bachelors degrees as well as masters degrees, and is considered primarily a teaching university. It is located in a large, urban area with a variety of ethnic backgrounds, and the principal industry in the area is computers and electronics.

As a part-time instructor at this university, the researcher had access to student rosters, grade sheets, and phone numbers. The students who fell under the guidelines outlined below were initially contacted by telephone, told of the research and asked if they would volunteer to participate in the study. The volunteers then met with the researcher on campus to be more fully informed of the research and their rights as participants. The names of each subject were only known by the researcher and were kept confidential.

The students used in this research had completed a year (two semesters) of calculus during the 1993-1994 school year. At the time of this study, there were only eleven students who had taken both semesters of Harvard Calculus. Of those eleven, only six had earned a grade of $\mathrm{B}+$ or better for both semesters. Since this study was to focus on the understanding of good calculus students, and to keep the number of students from each group equal, only six students were chosen from each type of class.

The six Harvard Calculus students were from two different sections and there were three students from each of the sections. The two sections were taught with different professors using the same text, Calculus (Calculus Consortium at Harvard, 1994). In one class, the software Xplore was used to generate and examine graphs and data. In the other Harvard class, computers were not used but it was suggested to the students to use a graphing calculator.

The six traditional students were from three different sections, so three professors were represented in that group. No technology was required, but some students chose to use calculators. The text used was Calculus, 2nd edition (Stewart, 1991).

Participants were interviewed twice, on campus, with various questions about their feelings about the course and on the key topics of calculus. Each interview took approximately 50 minutes, with no noticeable time difference between groups. The first 
round of interviews was done in September of 1994, while the followup interview took place in October 1994.

In the Harvard group, the age of the subjects ranged from 19 to 33 , with the average age being 23. The six subjects, consisting of four females and two males, were born in 6 different countries: Vietnam, South Africa, Peru, the Phillipines, India, and the United States. The group consisted of one mathematics major, one engineering major, one biology major, one chemistry major, and two computer science majors. During their first semester of Harvard Calculus in the fall of 1993, their grades were as follows: three A's, an A- and two B+'s -- that yields a 3.72 grade point average. For the second semester they earned two At's, an A, two A-'s and a B+, which translates to a 3.78 grade point average.

In the traditional group, the range of ages went from 18 to 42 , with an average of 24. There were four females and two males, with two subjects from the United States, one from China, one from Vietnam, one from India, and one born in Hong Kong. The group consisted of the following majors: civil engineering, electrical engineering, environmental studies, two computer science majors and a double major of mathematics and psychology. During their first semester of traditional calculus, the group as a whole earned four A's and two B+'s. For the second semester they earned an A+, three A's and two A-'s. Those grades translate to respective grade point averages of 3.77 and 3.90 , so there were small differences in grades between the Harvard and traditional groups for both semesters.

The initial meeting with the subjects consisted of an approximately 10 minute interview, which was audiotaped, followed by a written test. The second meeting was almost all interview, which again was audiotaped, with a few questions given in writing.

The interviews and tests were designed to find out how well the subjects understood some of the key concepts in calculus. The topics they were tested on were functions, limits, continuity, differentiation, and integration. Some of the questions were computational, but the majority were conceptual in nature. There also were some questions in the initial interview regarding the subjects' opinions of the class and text, and how the class compared to other mathematics courses they had taken. The questions came from three sources: the researcher, the thesis committee, and the research that preceded this report (see Amit \& Vinner (1990); Dreyfus \& Vinner (1982, 1989); Ferrini-Mundy \& Graham (1994); Selden, Selden \& Mason (1994), and Williams (1991)). 
The following are the questions from the initial interview and test. Each numbered question below has a prefix of either $Q$ for question or $T$ for test. This will aid the reader when the questions are referenced later in this report.

INITIAL INTERVIEW

BACKGROUNDDATA

Name

Survey ID

Age

Sex

Major

Native Country High School Date Graduated

Highest High School Math Course First College Math Course

Grade in Calculus 1 Completed with Dr.

Grade in Calculus II Completed with Dr.

\section{INTERVIEW QUESTIONS}

Q1) On a scale of 0 to 10,0 being strongly negative, 5 being neutral and 10 being strongly positive, where would you place your overall feelings towards mathematics?

Q2) Do you find calculus to be less difficult, as difficult, or more difficult than previous math courses you've taken? Explain.

Q3) Was the first year of calculus what you expected it to be? Explain.

Q4) Do you see any real world usefulness of calculus, or do you see yourself using calculus in the future?

Q5) How does the instruction differ from other math courses?

Q6) How does the text differ from other math texts? 
Q7) What do you feel are the main topics or key subjects of calculus?

Q8) Can you tell me what a derivative is?

Q9) Can you explain the relationship between a derivative and a limit?

Q10) I'm going to show you 9 graphs, labeled G1-G9 (see below). Please sort the graphs into two piles; if the graph could be the graph of a function, place it in one pile; if not, place it in a separate pile. Explain your criteria for deciding whether or not a graph is a function. What is a function? Please note that each small square on the graph represents a 1-by-1 unit square.

\section{Graph $G_{1}$}

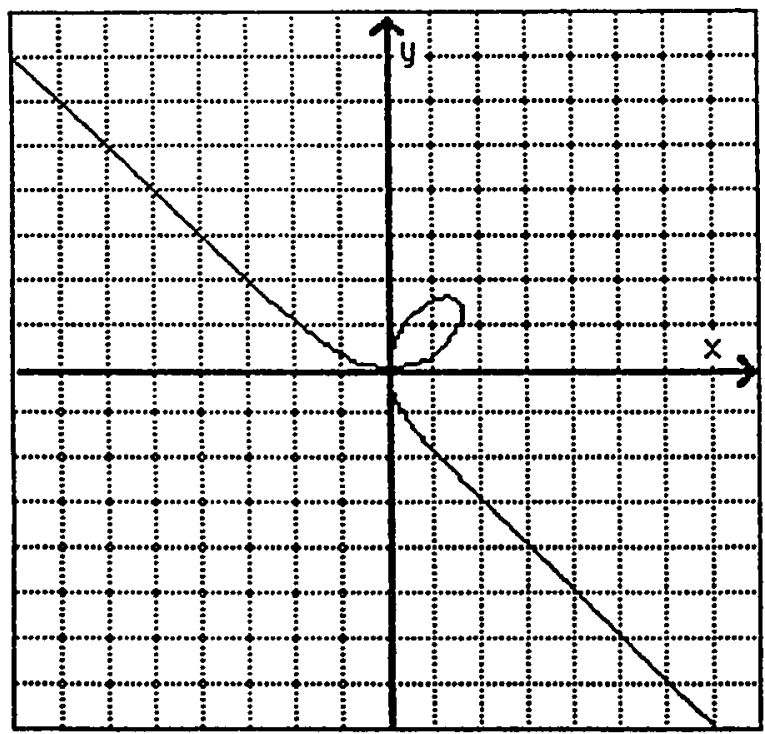

Graph G2

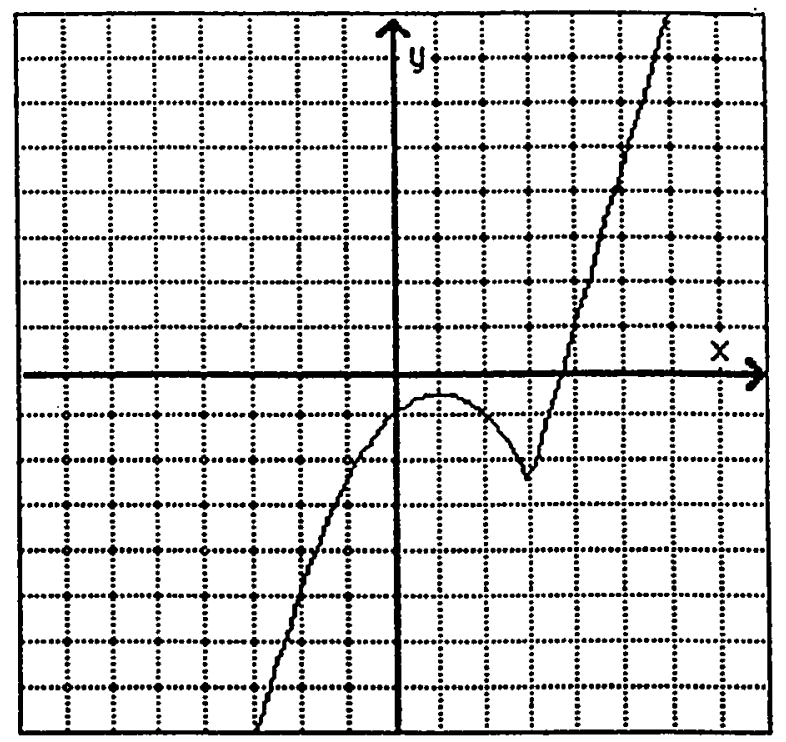



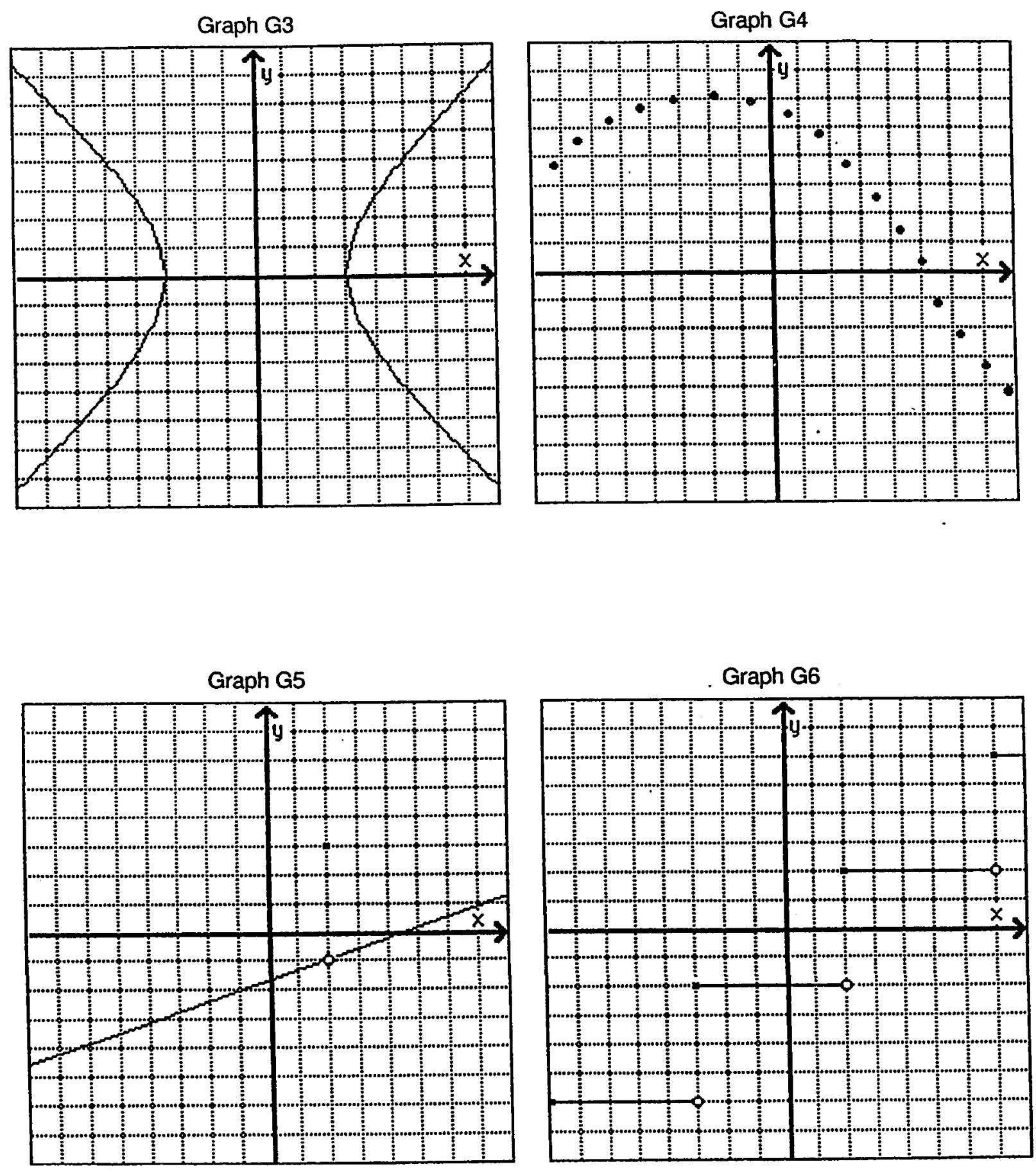

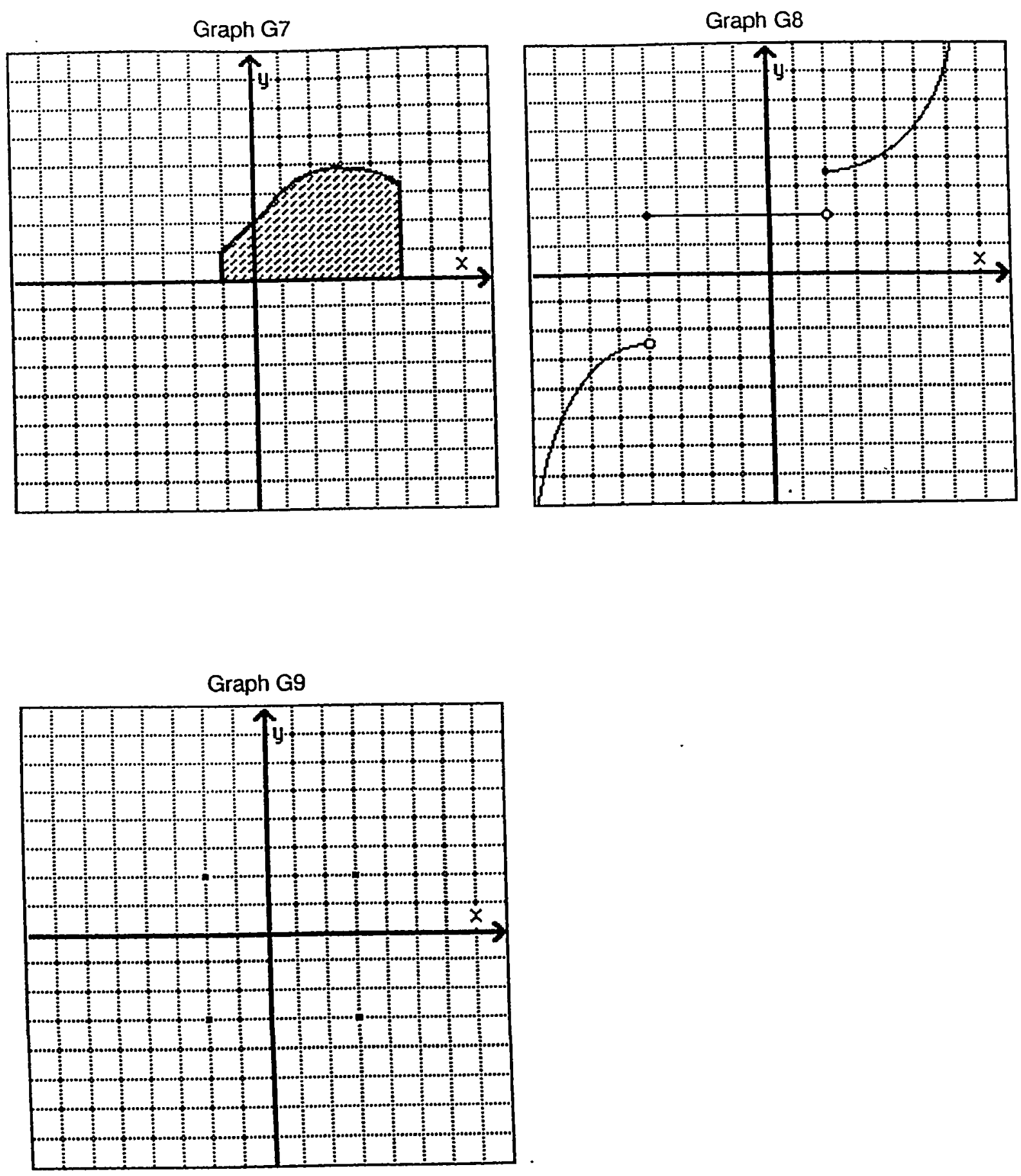
Q11) The table below is a partial table of values for some unknown function $f$. Assuming the function is differentiable at 3 , is it possible, with the given information, to approximate $f^{\prime}(3)$ ? If so, then approximate $f^{\prime}(3)$ and explain how you did so; if not, explain why not.

\begin{tabular}{|c|c|c|c|c|c|c|c|c|}
\hline$x$ & 2.8 & 2.9 & 3.0 & 3.1 & 3.2 & 3.3 & 3.4 & 3.5 \\
\hline$f(x)$ & -1.325 & -2.291 & -3.300 & -4.351 & -5.443 & -6.576 & -7.750 & -8.963 \\
\hline
\end{tabular}

This was the end of the audiotaped portion of the initial interview. As you can see, most of the interview was gathering background information on the subject and getting their feelings about mathematics and their calculus class. The last few questions of the interview, as well as the written test below, explored their level of understanding of functions, limits, continuity, and derivatives.

\section{WRITTEN TEST}

For this portion of the test, please feel free to use scratch paper, draw diagrams, use your calculator, or any other reasonable method to help you solve these problems. If you are not sure what the question is asking, please ask me. Also, if you are not sure of an answer, please do not guess -- simply write "Not Sure" under the question. Thank you for participating in this study.

T1) For the following questions, find the limit if it exists. If it does not exist, write $\mathrm{n} / \mathrm{a}$ in the space provided. If you are not sure, write NOT SURE. As stated earlier, the small boxes on the graphs represent 1-by-1 unit squares (see graphs on pages 14-16).

a) In graph G2, the $\lim _{x \rightarrow 3}$ is :

b) In graph G5, the $\lim _{x \rightarrow 2}$ is:

c) In graph G8, the $\underset{x \rightarrow 2}{\lim }$ is: 
T2) Suppose the graph below is the graph of $y=f(x)$. By examining the graph, find the following (if there is not enough information on the graph, simply write n/a in the space provided):

a) The precise intervals where $f(x)$ is increasing

b) The $x$ and $y$ intercepts of $f(x)$.

$x$-int

$y$-int

c) Local minimum (approximate)

d) Local maximum (approximate)

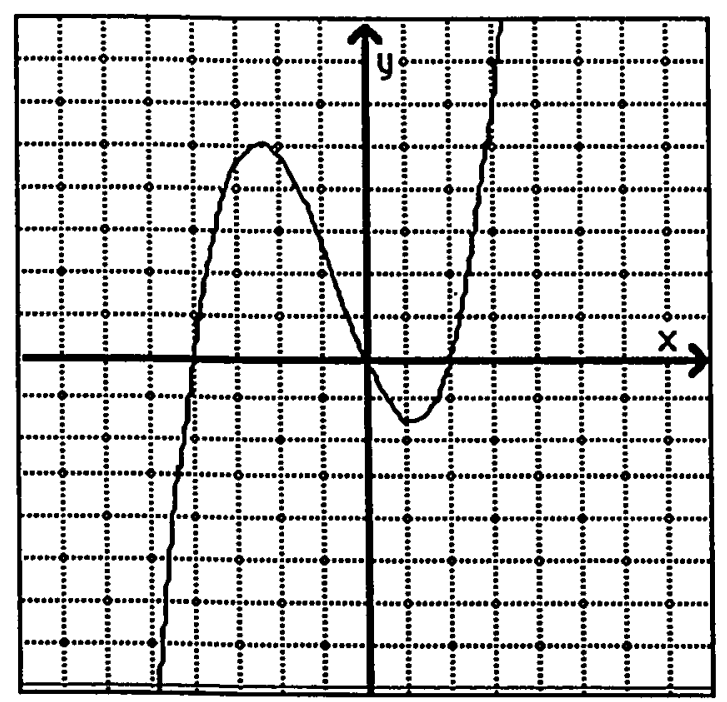


T3) Suppose the graph below is the graph of $y=g^{\prime}(x)$ (that is, it is the graph of the derivative of $\mathrm{g}$ ). By examining the graph, please find the following information for the function $g(x)$ (if there is not enough information on the graph, simply write $n / a$ in the space provided):

a) The precise intervals where $g(x)$ is increasing

b) Local minimum (approximate)

c) Local maximum (approximate)

d) On the axes below, sketch a graph of what the original function might look like.

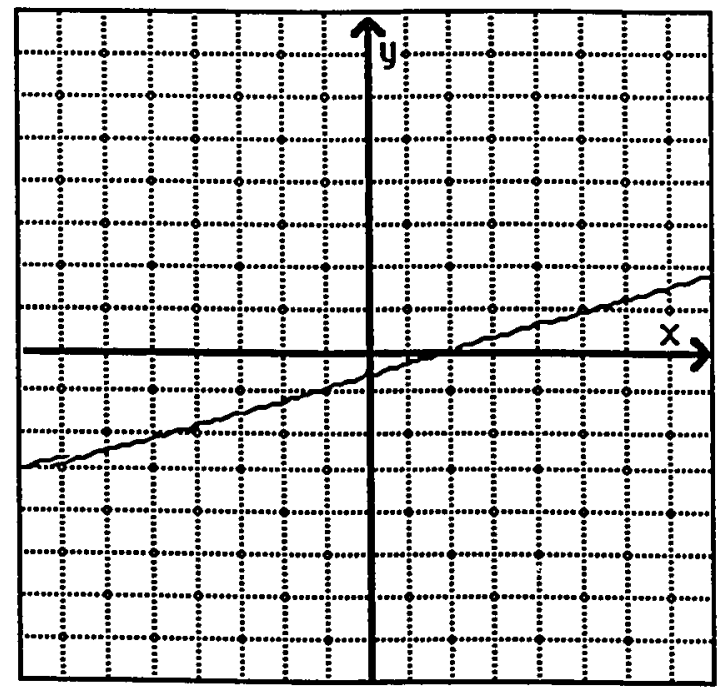


T4) On the graph below, the line $L$ is tangent to the graph of $y=f(x)$ at the point $(5,3)$. Find the following:
a) Find $f(5)$
b) Find f'(5)

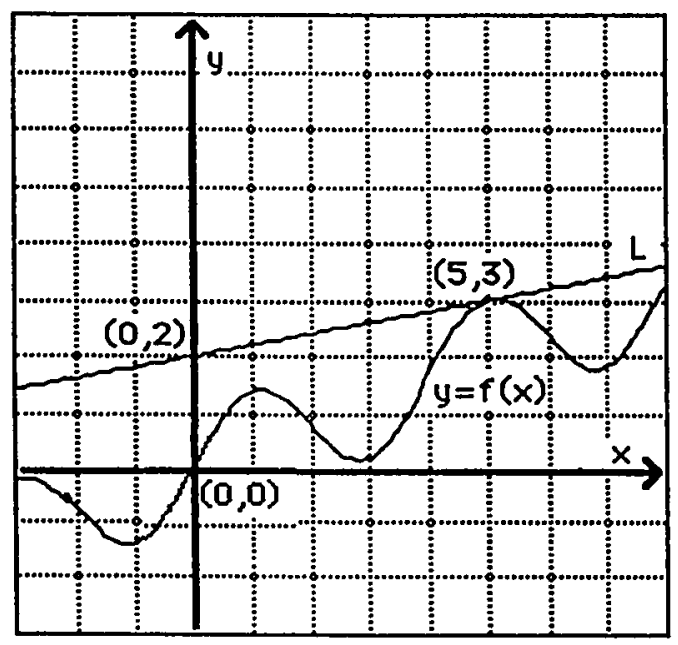

T5) Let $f(x)= \begin{cases}a x & x \leq 1 \\ b x^{2}+x+1 & x>1\end{cases}$

Find $a$ and $b$ so that $f$ is differentiable at 1 .

a

b

As the subjects were taking the test, it was stressed to them that they should not guess if they did not know the answer. The subjects were encouraged to ask questions about anything they were unsure of, and to use scratch paper, a calculator, a straight edge, or anything else that would help them with their problem solving. All necessary materials were provided.

After the interview and test, their tests and scratch paper were gathered and labeled with an identifying code (T1 through T6 were used for the subjects who took traditional calculus, $\mathrm{H} 1$ through $\mathrm{H} 6$ for the Harvard group). The cassette tapes were also labeled with the same code. For confidentiality purposes, the only thing labeled with 
the subjects' names was the initial interview form, which was only seen by the researcher. The subjects were later given pseudonyms to protect their identity. For easy group identification, the students from the traditional group were given names beginning with the letter $T$, while the Harvard group was given names beginning with the letter H. Soon after the initial interview, the audiotapes were transcribed and their responses were analysed.

\section{FOLLOWUP TEST}

The followup test was designed to touch on topics that either were not addressed in the first test or were asked about but in a different representation. The followup test was written as a script and was a little more flexible than the original test, since some of the followup had to be individualized depending on students' responses to the original test and their responses during the followup itself. The questions were not necessarily asked in the numerical order seen below; the questions are numbered with a prefix of $F$ for followup.

\section{FOLLOWUP SCRIPT}

F1) First I want to clarify your criteria for determining functions. You originally said that you used the to determine if a graph represented a function. Please explain.

F2) Originally, you said that were not functions. How would you change these graphs in order for them to represent functions?

F3a) Sketch a function $f$ such that $f(2)=3$ and $f(1)>f(5)$.

F3b) Can you give me an idea of what the derivative of the function you have drawn would look like?

F4) Can you tell me what it means for a function to be continuous? 
F5) I'm going to hand you a stack of cards labeled i, ii, iii, and iv (see below). On each of these cards there is a piecewise function.
i) $f(x)= \begin{cases}x+3 & x \leq 4 \\ 4 x-9 & x>4\end{cases}$
ii) $f(x)= \begin{cases}2 x-3 & x \leq 5 \\ 2 x+3 & x>5\end{cases}$
iii) $\quad f(x)= \begin{cases}\frac{1}{5} x^{2}+x-7 & x \leq 5 \\ x-2 & x>5\end{cases}$
iv) $f(x)= \begin{cases}\frac{1}{2} x^{2}-x-7 & x \leq 4 \\ 3 x-15 & x>4\end{cases}$

a) Identify which of the 4 piecewise functions are continuous.

b) Which ones are differentiable?

F6) Here are the graphs of those same four functions.
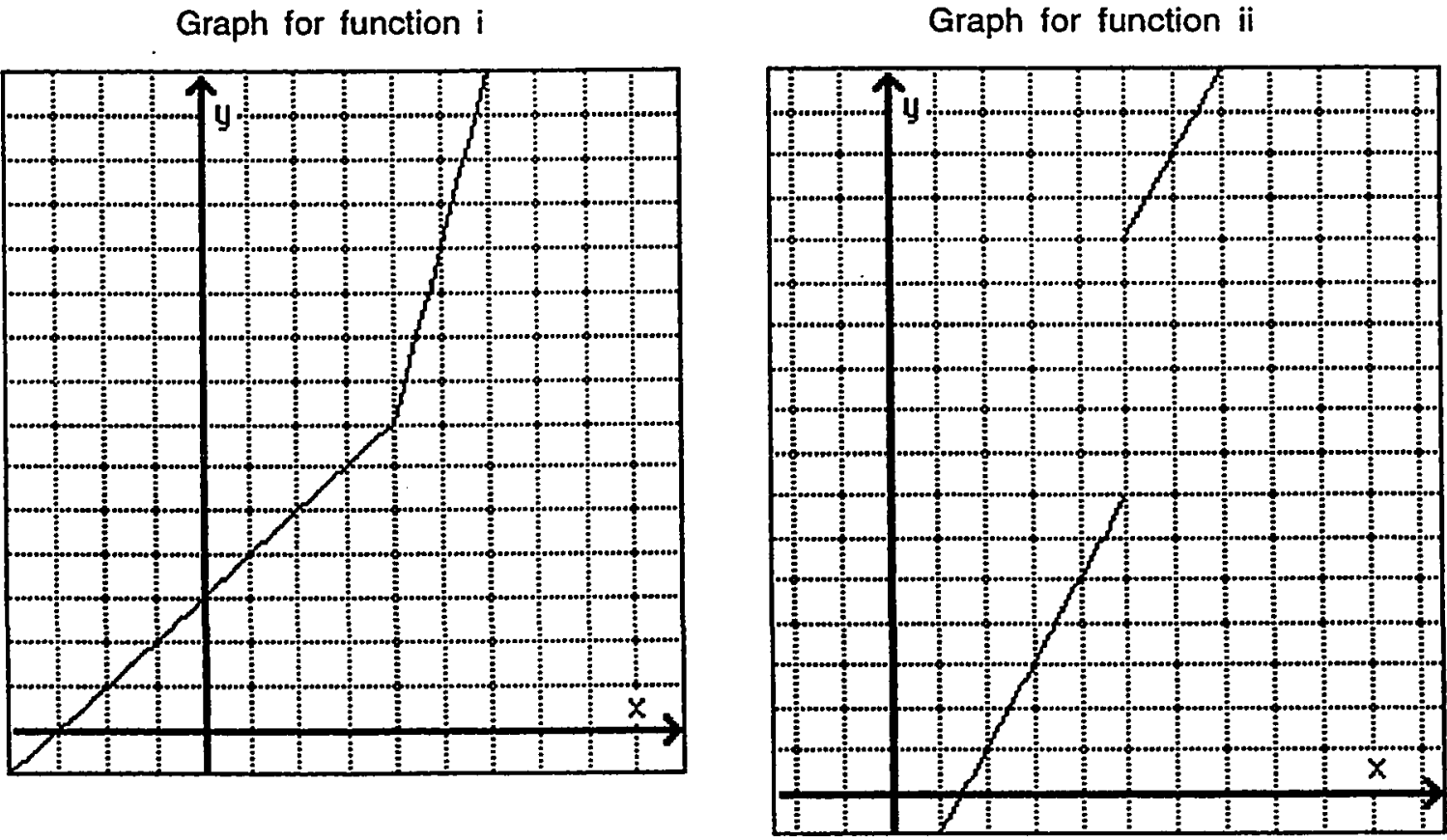
Graph for function iii

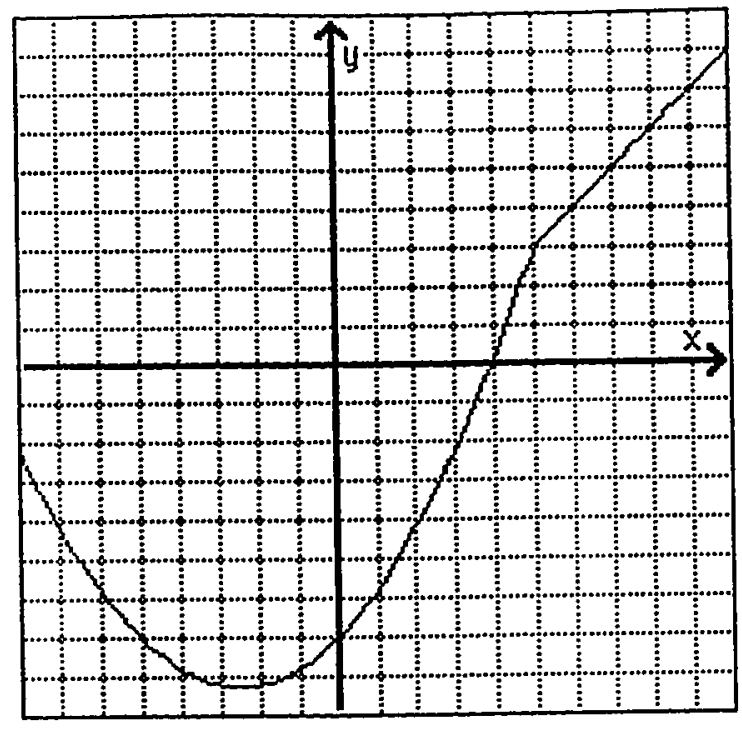

Graph for function iv

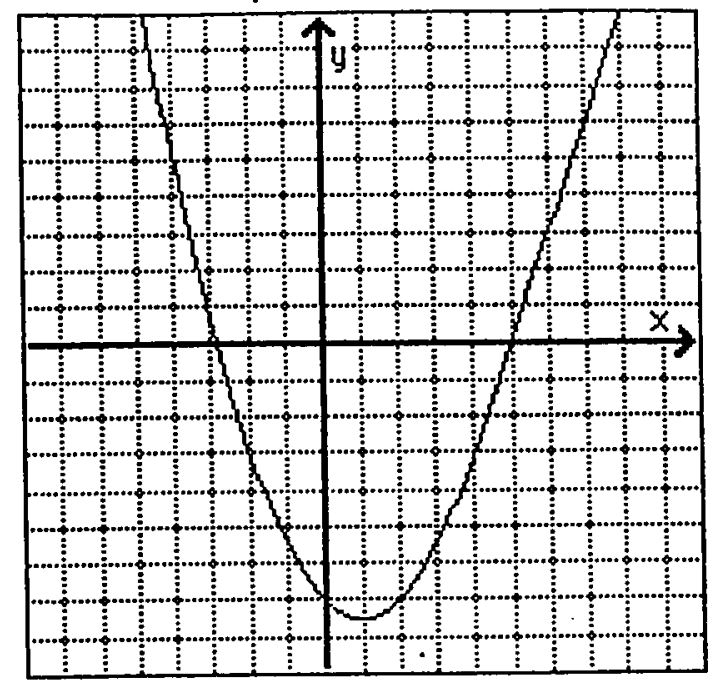

a) Now that you see the graphs, which ones do you consider continuous?

b) Which ones are differentiable?

F7a) If a function is continuous at $x=2$, must it be differentiable at 2 (that is, must the derivative exist at 2)? Sketch an example or counterexample.

F7b) If a function is differentiable at $x=5$ (that is, the derivative exists at 5 ), must it be continuous at 5 ? Sketch an example or counterexample.

F8a) Without actually finding the derivative, that is, without using any techniques of derivation, how would you estimate $f^{\prime}(2)$, where $f(x)=x^{x}$ ? You need not actually attempt to estimate the derivative, just explain the procedure.

F8b) Now can you tell me what $f^{\prime}(x)$ is explicitly? This time feel free to use any of the techniques you learned.

F9) On the original test you were given the graph of the derivative of a function and you were asked to sketch what the original function might look like. You have drawn a 
parabola and what I want to know is, is that the only function that would have that line for the graph of its derivative? In other words, is your solution unique?

Or, if they did not do the original problem correctly, ask: If the graph of the derivative of a function is a line, what would the graph of the original function look like?

F10) On the first test you were asked to find some limits, if they existed, by examining the graphs of some functions. Please explain each answer.

I'm going to give you the following limit questions for you to answer in writing. After you have answered them, please explain each answer. (The subject is then handed a sheet with the limit problems seen below.)

F11) Find the following limits (if they exist). If the limit does not exist, write $n / a$ in the space provided. You will be asked to explain your answers.

a) $\lim _{x \rightarrow 2} f(x)$, where $f(x)= \begin{cases}3 x+5 & x<2 \\ 3 x+5 & x>2\end{cases}$

b) $\lim _{x \rightarrow 3} f(x)$, where. $f(x)=\frac{3 x^{2}-9 x}{x-3}$

c) $\lim _{x \rightarrow-\infty} f(x)$, where $f(x)=\frac{3 x^{2}+2 x+1}{x^{2}-5}$

d) $\lim _{x \rightarrow 6} f(x)$, where $f(x)= \begin{cases}3 x-5 & x \leq 4 \\ 2 x+3 & x>4\end{cases}$

e) $\lim _{x \rightarrow 4} f(x)$, where $f(x)= \begin{cases}2 x+3 & x<4 \\ 5 & x=4 \\ x^{2}-5 & x>4\end{cases}$ 
F12) A student was given a function $f$ and asked to find the limit as $x$ approaches 0 . He plugged in numbers on each side of 0 and made the following table:

$\begin{array}{rl}x & f(x) \\ -.1 & 0.9 \\ -.01 & 0.99 \\ -.001 & 0.999 \\ -.0001 & 0.9999 \\ -.00001 & 0.99999 \\ -.000001 & 0.999999 \\ -.0000001 & 0.9999999\end{array}$

$\begin{array}{rl}.1 & 1.1 \\ .01 & 1.01 \\ .001 & 1.001 \\ .0001 & 1.0001 \\ .00001 & 1.00001 \\ .000001 & 1.000001 \\ .0000001 & 1.0000001\end{array}$

a) What can you conclude about the limit of the function $f$ as $x$ approaches 0 ?

b) Would your answer change if you were told that $f(0)=7$ ?

c) What could you say about the limit if 0 is not in the domain of $f$, that is, $f(0)$ is undefined?

F13) If for some function $g$, the limit as $x$ goes to 3 is 5 , could we conclude that $g(3)=5$ ? Explain your answer or sketch an example or counterexample. 
F14) By examining the graph, find $\int_{1}^{4} f(x) d x$.

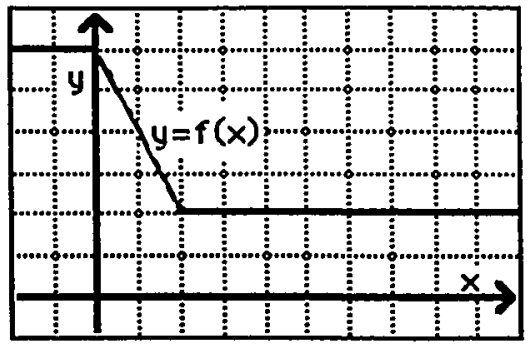

F15) Find the equation of the line tangent to $y=x^{2}$ at the point $(3,9)$.

After the second tests were completed, the audiotapes and paperwork were again labeled with the appropriate identifying code. The tapes were then transcribed, and the responses were analyzed. The results of the two tests were then combined and examined in two different ways. First, there was a student assessment, and secondly, there was a question-by-question arrangement of the results.

In the student assessments, each student was assessed on his/her overall understanding of function, limit, derivative, continuity, and integration. A brief paragraph was written on each topic for each student. There were also some brief comments on his/her general attitude and performance. When reading the student assessments, the reader may notice that there are not a lot of direct quotes or excerpts from the students' work. This was done intentionally, as the assessments are meant to simply give the reader an idea of how well each student comprehends each topic; it is not the purpose of the assessments to detail every response the student made on a particular topic. The majority of the subjects' work will be seen in the question-by-question results.

For the question-by-question results, the questions were categorized by topic. For example, all questions pertaining to derivatives were grouped together, with questions then put together under subcategories, such as estimating derivatives or interpreting the graph of a derivative. After they were categorized, each question was examined to see how each student group responded and approached that particular question. This made it relatively easy to see any differences between the Harvard Calculus and traditional calculus groups. This second approach to arranging the questions also revealed some interesting similarities, which are included in the next chapter. 


\section{CHAPTER 4}

\section{RESULTS}

As stated in Chapter 3 , the results of the two tests were compiled in two different ways. First, each student was assessed on his/her understanding of the key topics of calculus, and secondly, the groups' responses were compared question-by-question. We will begin with the student assessments.

\section{STUDENT ASSESSMENTS}

For the student assessments, the subjects' responses were analyzed to determine how well they understood each topic. This was not an easy task, since it.was often the case that a subject would answer a couple of questions on a topic perfectly, and yet later answer questions on the same topic inaccurately or sometimes even contradict earlier statements. Sets of related questions helped determine the subjects' depth of understanding for each of the concepts; however, this sometimes required subjective judgements.

In describing the students' understanding of each concept, an overall qualitative judgement was made. A common remark made throughout the student assessments is that the subject gave an acceptable definition. The reader should be aware that this does not mean the student gave a mathematically formal definition of that particular concept. In fact, no student ever offered a formal definition when defining a concept. An informal but accurate description of the topic in question was considered an acceptable definition. For example, defining continuous functions as being those functions that can be sketched without lifting one's pencil was acceptable (even though this is not entirely accurate). The assessments start with the six students from the traditional group.

\section{TRADITIONAL STUDENTS}

\section{Tracy}

General Comments: Tracy felt that calculus is more difficult than previous mathematics courses. She felt that the instruction was the same as other mathematics courses, and she also commented that the book did not help her much. "I learned from the teacher, not 
the text." She did very well on both interviews.

Function: Tracy gave a very strong performance on all questions pertaining to functions. She gave an acceptable definition of function, saying that a function is when "there is a unique value of $y$ for any $x^{\prime \prime}$ and she had a valid criterion for determining functions. Tracy correctly identified all functions and nonfunctions. She was also able to interpret the graph of a function, finding the $x$ and $y$ intercepts, local minimum and maximum, and where the graph was increasing. In the followup, she had no problem explaining how to change the nonfunctions to functions, and she had no problem sketching the graph when given specific parameters (problem F3a).

Limits: She answered virtually every question on limits correctly.

Continuity: Tracy did very well in this area. She gave an acceptable definition, was able to correctly identify which functions were continuous both algebraically and graphically, and showed understanding of the relationship between continuity and differentiability.

Derivatives: Tracy gave an acceptable, although somewhat limited, definition of derivative, calling it a "rate of change," and correctly identified the graphs of differentiable and non-differentiable functions. She understood that differentiability implies continuity, and not the converse. Tracy gave acceptable answers to the numerical and algebraic derivative estimation problems, although she did make an odd remark during her response to Q11; she said that it would only be possible to estimate the derivative if she knew the graph was not fluctuating, and then added that "it depends on how much around 3 [the function] is continuous." She could not differentiate $y=x^{x}$, although her answer, $\left(\mathrm{xx}^{\mathrm{x}-1}\right)$, was the most common response in both groups of students. Her biggest mistakes occurred on the graphical derivative questions (problems T3 and T4). For problem T3, she thought that both the $x$ and $y$ intercepts of the graph of the derivative told where a critical point is; this caused her to sketch the original function inaccurately. On problem T4, the problem which involves finding the slope of the tangent line to solve for the derivative, she (unnecessarily) found the equation of the line and then substituted 3 into the equation to get the wrong answer, a common mistake. 
Integration: She accurately answered question F14, the graphical integration problem, but as stated above, she did not sketch the graph accurately in problem T3, part $d$.

\section{Tylinn}

General Comments: Tylinn felt calculus was more difficult because she found it to be more theoretical. She said in calculus "you think about math in different ways than high school." She found the book to be "too technical." Tylinn has a fairly shallow understanding of many of the key topics in calculus.

Function: She did well on everything pertaining to functions except a definition. She defined a function as any equation with variables in it.

Limits: She understands that the value of a function at a point and the limit at that point can be two different things, and she is aware that the right and left limits need to be equal for the limit to exist. However, she feels that in order for a limit to exist at point a, a must be in the domain of the function. Tylinn also demonstrated that she does not understand one-sided limits when presented in a graphical context. For instance, looking at graph G5 (see page 17), when asked what the right hand limit is as $x$ goes to 2 , she answered 3 (the value of the function at the singleton) stating that " $x$ is like a line here, a vertical line here, and if you approach from the right you get this point [points to $(1,3)] . "$

Continuity: Tylinn recognized continuous functions graphically, but could not do so algebraically with the piecewise functions. She did not verbalize an acceptable definition, although she clearly believes the graph of a continuous function should not have any breaks or gaps in it. She does not understand the relationship between continuity and differentiability.

Derivatives: Tylinn's definition of derivative was acceptable but it lacked depth. She said the derivative "tells you where the graph is going, whether it is increasing or decreasing." However, she answered problem T4 correctly on the written test, so she must understand that the derivative is the slope of the tangent. She did poorly on the estimation problems, and she could not differentiate $y=x^{x}$. Tylinn thinks that differentiability does not imply continuity, but does believe that continuity implies 
differentiability. She could not recognize differentiable functions either algebraically or graphically. Given the graph of the derivative, she could not accurately sketch the original function, did not accurately interpret information from the derivative, and she believes that the graph of the original function is unique up to horizontal shifts. She also answered incorrectly when asked to find the equation of the line tangent to $y=x^{2}$ at $(3,9)$. Overall, she has a poor understanding of derivative.

Integration: Tylinn answered problem F14 correctly, but again, she missed problem T3, part d.

General Comments: Ted found calculus to be "less difficult than trigonometry, but more difficult than algebra." He thought the book was "more abstract" but "fairly readable." He felt that the main difference in instruction was that the course "goes by a lot faster."

Eunction: Ted gave a good definition of function and he identified every graph correctly. Every question he answered about functions was answered correctly, although he did forget to answer one question on $x, y$ intercepts from the first test.

Limits: He did very well on every question about limits. He initially missed two of the graphical limit questions, but he corrected both mistakes in the followup session.

Continuity: He gave an acceptable definition (after some initial confusion), identified continuous functions both algebraically and graphically, and showed understanding of the relationship between differentiability and continuity.

Derivatives: Ted gave a correct definition of derivative, calling it "the slope of a tangent line to a curve," and did very well on every question about derivatives except the following: he did not recognize the differentiable functions either graphically or algebraically, and he could not differentiate $y=x^{x}$. It should also be noted that when he answered problem T3 of the first test, he first took the antiderivative of the equation of the line to get an idea of what to sketch.

Integration: He answered problem F14 correctly, and he sketched an appropriate graph 
for question T3, part d.

Tanya

General Commenis: Tanya felt that calculus was no more difficult than her previous mathematics courses, and she saw no difference in the style of instruction other than an improvement in quality. Tanya did poorly on a number of questions, and was the weakest student from the traditional group.

Function: Although she could not define function, Tanya does have a fairly solid understanding of what a function is. She recognized all functions and gave a valid criterion for doing so; her one mistake on recognition was thinking G7 (the shaded region) was a function. Her major weakness seems to be actually working with functions and their graphical representations. She could not answer three of the limit questions because the functions were piecewise functions. "I remember what piecewise functions are" she said "but I don't remember how to do them." Also, when answering a question on derivatives she attempted to sketch a third degree polynomial but her sketch more closely resembled a fifth degree polynomial. For problem T3, part $d$, she derived a 2nd degree polynomial but her sketch looked nothing like a parabola (see page 75 for her diagram). This is an indication that she is much more comfortable with and adept at working with functions in an algebraic representation, and represented by no more than one equation.

Limits: Tanya also has a poor understanding of limits. For the graphical limit questions she said the limit at the point in question is simply the value of the function at the indicated point. As stated above, she did not answer the limit questions involving piecewise functions, but she was able to answer both of the limit questions that involved rational functions (question F11, parts $\mathrm{b}$ and $\mathrm{c}$ ), so she does have some procedural skills. Perhaps the biggest indicator of her poor understanding of limits was seen when she was answering one of the limit questions; "the limit at 2 is [one value], [but] as it approaches 2 it's something different," so she is clearly confused on limits -- perhaps the language of limits is the problem. Unfortunately, she was not asked the numerical limit question. 
Continuity: Tanya believes that in order for a function to be continuous it must pass the horizontal line test. She could not give any reasonable definition of continuity and missed every question about continuity. She has a very poor understanding of continuity.

Derivatives: When asked what a derivative is, she initially began to describe the process of differentiating a polynomial. After some thought, she then stated that "the first derivative is like equal to . . . like when you're graphing, [the] first derivative is equal to a minimum point of a parabola or something. I know [the derivative] is like the limit as $x$ approaches infinity of the function." In addition to her inability to define derivative, Tanya could not answer either estimation problem, could not sketch the original function given the graph of the derivative, has no idea how to recognize differentiability, does not understand the relationship between differentiability and continuity, and missed almost every question about derivatives. It should also be pointed out that for the graphical derivative questions (problems T3 and T4), she tried to convert the problem to an algebraic representation before answering the question. She then usually made an error in her algebra or in her derivations so her answer was still incorrect.

Integration: As she did for the graphical derivative problems, Tanya attempted problem F14 algebraically. Even though she answered incorrectly due to an error in her algebra, her method was acceptable, although not the easiest approach. She was not able to sketch a solution for problem T3, part d, correctly.

\section{Tarnmy}

General Comments: Tammy felt that calculus was less difficult than previous mathematics courses because "if you understand the basics, it just builds on that." She did not see any major differences in instruction except "in college, you have to figure things out on your own." Tammy has a much stronger command of procedures than she does understanding of the concepts.

Function: Tammy feels that continuity is necessary for functions. Because of this, she made several mistakes when attempting to identify functions graphically. She was able to sketch a function with the given parameters and she answered problem T2 on the first test correctly. 
Limits: Procedurally she is sound, but Tammy is tied to the idea that if $f(a)=b$, then the limit at a must also be $b$. This caused her to miss several questions.

Continuity: Tammy did not verbalize an acceptable definition, but she clearly thought of continuity as having no breaks or gaps. And although she did not identify the continuous functions algebraically, her method was acceptable (she tried to graph them, but she did a poor job of it). She was able to identify the continuous functions when they were presented to her graphically. She feels that a function is continuous if and only if it is differentiable.

Derivatives: Tammy defined derivative by saying it is "the slope of the tangent," but her skills at interpreting the graph of a derivative and estimating derivatives are weak. Also, she did not recognize differentiable functions graphically or algebraically. On the positive side, she was procedurally strong; she successfully found the derivative of $y=x^{x}$, and she was able to find the equation of the tangent line for problem F15.

Integration: She correctly answered question F14, but did quite poorly at interpreting the graph of the derivative and sketching the original function given the graph of the derivative.

\section{Tuong}

General Comments: Tuong felt that calculus was "much more difficult" than previous mathematics courses. He said that although he saw some applications of calculus, he did not "see any use in it." The main difference he saw in the instruction was the speed of the material. He also said at one point that "with math understanding it and explaining it is different from actually doing it." Tuong needs to have his functions represented algebraically, as his tendency was to convert a graph to an equation before attempting any work.

Function: Tuong feels that functions must be continuous, and his definition "plug in a number, and out comes a $y^{\prime \prime}$ definitely lacks depth. Even with this poor idea of what functions are, Tuong did well on most questions pertaining to functions. As stated above, Tuong revealed a strong preference for the algebraic representation of functions. 
Limits: Tuong has a poor understanding of limits. He does not understand the necessity of the right hand limit equaling the left hand limit in order for the limit to exist. He also believes that if $f(a)=b$, then the limit at $a$ is $b$. He was also inconsistent, saying at times the limit at a does not exist if $a$ is not in the domain of the function, and other times saying that is irrelevant.

Continuity: At first he gave the typical "you can draw it without lifting your pencil" definition, but then he changed it by saying that a function is continuous as long as the domain has no breaks or gaps. Because of this, he could not identify continuous functions either in algebraic or graphical form. He does not understand the relationship between continuity and differentiability, thinking that a function is continuous if and only if it is differentiable.

Derivatives: Tuong could not define derivative, could not do the estimation problems, and generally did poorly on every question pertaining to derivatives. His sketch for problem T3, part d, was fairly accurate, but he needed to estimate the equation of the line first and then take the antiderivative of that. Because of his estimate, his sketch was off a bit.

Integration: He answered problem F14 correctly, but he again showed a tendency to have the problem set up algebraically. As stated above, he also sketched an acceptable graph for problem T3.

\section{SUMMARY OF TRADITIONALSTUDENTS}

In summary, the traditional group did well on functions and limits, but seemed a little weak on the other topics. The table on page 44 summarizes all twelve students' understanding of the topics explored in this study. For analysis of these results, see Chapter 5. 


\title{
HARVARD STUDENTS
}

\author{
Helen
}

General Comments: Helen found calculus more difficult partially because she did not know what to expect. She stated that in her class there was "a lot more description of how you came to the conclusions that you came to, instead of just a number answer. A lot of word descriptions." She also said that the book was "in some ways much more vague" than other mathematics texts.

Function: Helen said a function is "when given inputs, there is only one output," and her criterion for recognizing a function was good. For recognition, she missed only one graph, thinking G7 (the shaded region) was a function. She sketched the function with the given parameters (problem F3a), changed nonfunctions to functions, and she correctly identified information from the graph of a function (question T2).

Limits: Helen is weak procedurally, missing 4 out of 5 of the procedural limit questions. She was not sure what it meant for there to be different one sided limits and was inconsistent if the limit at point a was different than the value of the function at a (graphically, she answered correctly; numerically and procedurally, she said the limit does not exist).

Continuity: She gave an acceptable definition of continuity, saying that the graph should have "no breaks or holes." Helen identified continuous functions graphically and her method for identifying continuous functions algebraically was good, although she did miss one due to an arithmetic error. The subject understood the relationship between differentiability and continuity.

Derivatives: She gave a good definition, saying that the derivative is the "slope of the graph of the function." Helen had acceptable responses to both estimation problems, and interpreted the graph of the derivative correctly (with one minor mistake, thinking on problem T3 that a maximum could occur at $x=-8$ ). When given the graph of the derivative she was able to sketch the original function correctly. She identified which function was differentiable graphically, but could not do so algebraically. She could also find the derivative given two points on the tangent line. Helen could not differentiate 
$y=x^{x}$, and she said she forgot how to find the equation of the line tangent to $y=x^{2}$ at $(3,9)$. Helen's primary weakness is procedurally-oriented questions.

Integration: Helen answered problem F14 correctly, and had no problem with question T3, part d.

\section{Henry}

General Comments: Henry felt that in calculus, the emphasis was on understanding, not just finding the right answer. He commented that in previous mathematics courses "you just looked at it and figured out some numbers" but "in calculus, you have to understand everything a lot more." However, the book "threw [him] off" because it has "no formulas, and there's no answers" in the back of the book. He also said "the process was more important than the answer; maybe not more important, but it was stressed."

Function: After some confusion, the subject came up with good criteria for determining functions. He ultimately missed only 1 of the graphs, G4. Discrete functions seemed to cause him some confusion, since he also could not change G9 into a function. The subject was able to identify $x$ and $y$ intercepts, local minimums and maximums, and other features of a function when the information was given graphically. He sketched the function with parameters, and with one exception (G9) was able to turn nonfunctions into functions.

Limits: Henry answered everything correctly about limits and seems to have a thorough understanding of the concept.

Continuity: He had a very good idea of what continuity is. Henry answered everything about continuity correctly, and even went so far as to say if a function is continuous "the limit coming from the left and right will be the same." The subject understands the relationship between differentiability and continuity.

Derivatives: The subject gave an acceptable definition and was able to do both estimation problems. He correctly answered every question pertaining to derivatives except the following: Henry could not recognize differentiable functions (regardless of how the functions were represented), he could not solve problem F15, he could not differentiate 
$y=x^{x}$ (although he did recall that you need to take the logarithm of both sides of the equation), and for problem T3, he thought that there could only be one unique function that could have the given graph of the derivative.

Integration: He answered question F14 correctly, and sketched an appropriate graph for problem T3, part d.

\section{Hannah}

General Comments: Hannah felt that calculus was just as difficult as her previous mathematics courses, and she said that Harvard Calculus was the first mathematics course that made her think of mathematics as "not just numbers" and it helped her "think more broadly." She also said that in previous mathematics courses she was simply given formulas, but in this course "my professor explained how a formula was derived." About the book, she said "other texts are like you follow a pattern and that's it. But here you have to keep thinking differently for each [problem]."

Function: The subject misidentified only one graph, and generally answered everything on functions correctly.

Limits: Hannah did poorly on limits. She missed 2 of the 3 graphical limit questions, she could not do any of the procedural limit questions, and answered the conceptual limit questions incorrectly.

Continuity: Hannah gave an acceptable definition of continuity, recognized continuity graphically, and knew how to recognize continuity algebraically (although she missed one due to an arithmetic error). Her only major error was that she believes continuity implies differentiability.

Derivatives: She defined derivative as "the slope of the curve"; however, it is clear that she thinks of the slope of the tangent as different from the slope of the curve, since for problem T4 she made the comment that the slope of the tangent is only approximately equal to the slope of the curve. Given the graph of the derivative, she was able to interpret it correctly and sketched the appropriate curve of the original function. She was able to find the equation of the line tangent to $y=x^{2}$ at $(3,9)$. Her estimation skills 
are a little weak, since for both of the estimation problems she suggested sketching the curve and estimating the slope of the tangent (she never suggested using the difference quotient). Hannah did not recognize differentiability (either graphically or algebraically) and does not understand that differentiability implies continuity. She could not differentiate $y=x^{x}$.

Integration: She could not answer problem F14, saying "I think you need to take the derivative or something." As noted above, she was able to sketch an appropriate graph for problem T3.

Herb

General Comments: Herb found calculus to be easier than he had expected. In fact, he thought it was even easier than previous mathematics courses, primarily because of his instructor. This is odd, though, because of all the Harvard students, Herb had the poorest understanding of the concepts. He felt the Harvard text was "easier to read [and] understand" than other mathematics books.

Function: Herb did poorly in every aspect. He gave a weak definition ("a certain formula that gives me only one result, not two"), he did not have a legitimate criterion for determining functions (saying that a graph represented a function if he remembered it from the class), and he did not identify any of the functions.

Limits: Herb did not make that many errors on limits, but the ones he did make were usually conceptual in nature. Also, he was very inconsistent. When asked questions that had the limit at a point not being equal to the value of the function at that point, he answered correctly when the question was posed graphically, incorrectly when the question was posed algebraically, and said he did not know in the case of the numerical question. He only missed 2 of the procedural questions in the followup interview, the one mentioned above plus part $b$, but this mistake was a big one since he answered " 0 because it's 0 divided by $0 . "$

Continuity: Herb gave an acceptable definition of continuity and identified the continuous functions both algebraically and graphically. He feels that continuity implies differentiability, but that differentiability does not imply continuity. 
Derivatives: Herb could not define derivative, although he did say he thought it was related to the slope. He did poorly interpreting the graph of a derivative, and he could not identify differentiability either graphically or algebraically. Herb also did poorly on the estimation problems (he could not do the numerical problem and he only suggested sketching the algebraic problem and estimating the slope). On the positive side, he was able to solve problem T4, and he drew a correct sketch of the function given the graph of the derivative.

Integration: He did not know how to solve the graphical integration problem (problem F14). He did, however, complete problem T3, part d, successfully.

\section{Harriet}

General Comments: The subject felt that Harvard Calculus was more difficult than previous mathematics courses because it involved more interpretation of information whereas "in other math classes, you just sort of follow formulas." She appreciated the use of the computer in the classroom, and felt that the computer helped with her understanding of the material.

Function: Harriet gave an acceptable definition of a function, and her criterion for identifying functions was "for every $x$ there's only one value of $y$." She correctly identified all graphs for problem Q10. She was able to change nonfunctions to functions, and sketched the function with the given parameters.

Limits: Procedurally, she is a little weak (she missed three out of five of the procedural limit questions). She understands that in order for a limit to exist, the left and right limits must be equivalent, but how she interprets left and right limits graphically is interesting; regarding graph G5, she said that the right hand limit is the value at the singleton point, namely 3 . "If you have a point up here, then that's where the function is approaching from that [the right] side. If you want me to explain it I can't (laughs)." Harriet also believes that the limit exists at point a only if 1) point $a$ is in the domain and 2) $f(a)$ is the point the graph is approaching from the left and the right.

Continuity: The subject gave an acceptable definition and could identify continuous functions graphically, but she could not do so algebraically. She understands that 
differentiability implies continuity and not the converse.

Derivatives: Harriet did very well on everything regarding derivatives. The only thing she missed was she could not differentiate $y=x^{x}$ and she could not identify differentiable functions algebraically (but no one could).

Integration: She answered problems F14 and T3d correctly.

\section{Hu-Anh}

General Comments: Hu-Anh felt that calculus was no more difficult than previous mathematics classes. "It seemed like regular math. I didn't think it was hard." However, she felt the instruction was much different. "In previous math [classes], all I had was 'here's a formula, plug in the numbers.' You don't see what the theory is behind it. In calculus, I saw real applications." Regarding the text, she said "in other books, they give you an example and then say 'here, do this.' In this book, if you understand the material, you don't need to see an example."

Function: She gave a very poor definition, saying a function is "something that depends on something," and her criterion for determining functions lacked depth; "if y depends on $x$, then it is a function." She missed 3 of the graphs, and it was clear that if she was not familiar with the graph, she was more likely to deem it a nonfunction.

Limits: She did not do very well with limits and she was inconsistent in her beliefs. In fact, during the first interview she answered question $Q 1$ by finding the area under the curve, but during the followup interview, she realized that was wrong. Hu-Anh has a poor understanding of one-sided limits as well, and she believes that in order for a limit to exist at some point a, a must be in the domain of the function (although she is inconsistent in this belief). Her method of solving the procedural limit questions was unorthodox. In fact, for two of the procedural limit questions, she simply substituted values close to the limit in question. On another one, she concluded that the limit is 1 because, as she put it, "infinity divided by infinity is 1." This revealed a serious flaw in her thinking.

Continuity: Hu-Anh could not verbalize a definition for continuity, but from her 
sketches, it was clear she believes a continuous function should have no breaks or gaps. She was able to identify continuous functions both graphically and algebraically. She knew that differentiability implies continuity, but she was not sure about the converse.

Derivatives: She gave an acceptable definition and was able to complete both estimation problems (she used the difference quotient on the numerical problem, and suggested sketching a tangent and solving for its slope for the second). She solved the equation of the tangent line, she was able to sketch a function given the graph of its derivative, and she was aware that her sketch could be shifted vertically. Hu-Anh could not differentiate $y=x^{x}$ explicitly, and she could not identify differentiable functions algebraically or graphically. She also missed problem T4 on the original test.

Integration: She answered questions F14 and T3 correctly.

\section{SLUMMARY OF HARVARD STUDENTS}

Overall, the Harvard group seemed to have a fairly good understanding of most of the topics. Limits was clearly the topic they understood the least; the procedural limit questions seemed to be more challenging for them than they were for the traditional students. For a quick look at all twelve students understanding of the five topics this study examined, see Table 1 on the next page. Analysis of these results will be seen in Chapter 5.

\section{CONCLUSION FROM STUDENT ASSESSMENTS}

After reading the student assessments, there are a few conclusions one can make about the two groups of students. First of all, the Harvard group seems to find the class to be more than just a list of rules and procedures. Several comments were made that in the Harvard class they did not simply "follow formulas," and that the ideas behind the formulas and procedures were more important than simply finding the right answer. Their comments on the textbook were similar to their comments on the class, such as Hannah's comment that the book does not merely ask the student to copy the examples. No such comment was made by any of the traditional students.

Secondly, the traditional group seemed to have a slightly better understanding of limits, although there was much confusion in both groups on one-sided limits and 
whether or not a point needs to be in the domain of the function for a limit to exist at that point. Neither group truly excelled on limits.

Finally, the Harvard group did much better than the traditional group when it came to answering questions on continuity and derivatives. On everything from defining the concepts, to estimating the derivative, to recognizing continuity and differentiability, the Harvard students had more success.

The groups' differences and similarities will be more apparent when looking at the results topic-by-topic. Analysis of and conclusions from the results are found in Chapter 5. Below is a table that provides a quick summary of the students' understanding of each topic. Their understanding was rated good, fair, or poor for each topic, and this rating required some subjective analysis of their responses.

TABLE 1: STUDENT ASSESSMENTS

\begin{tabular}{llllll}
\hline Student & Function & Limit & Continuity & Differentiation & Integration \\
\hline Tracy & Good & Good & Good & Fair & Good \\
\hline Tylinn & Fair & Fair & Fair & Fair & Good \\
\hline Ted & Good & Good & Good & Good & Good \\
\hline Tanya & Fair & Fair & Poor & Poor & Poor \\
\hline Tammy & Poor & Fair & Fair & Fair & Fair \\
\hline Tuong & Fair & Fair & Poor & Poor & Fair \\
\hline Helen & Good & Fair & Good & Good & Good \\
\hline Henry & Good & Good & Good & Fair & Good \\
\hline Hannah & Good & Poor & Fair & Fair & Fair \\
\hline Herb & Poor & Fair & Fair & Fair & Fair \\
\hline Harriet & Good & Fair & Fair & Good & Good \\
\hline Hu-Anh & Good & Fair & Good & Fair & Good \\
\hline
\end{tabular}




\section{TOPIC-BY-TOPIC RESULTS}

For the topic-by-topic results, each question was looked at separately, and each group's responses were tallied. Before the groups' responses were considered, however, appropriate answers had to be determined for each question. There were usually three types of answers for each question: A good answer, an acceptable answer, and an unacceptable answer. The main difference between a good answer and an acceptable answer is that an acceptable answer lacks the depth of a good answer, and is often not well stated. The more direct, straight-forward questions will only have good answers or unacceptable answers.

Below you will find all of the test questions arranged by topic. Answers, along with some additional comments, are found below each question. All remarks made by the interviewer, including the original test questions, appear in italics. The astute reader will notice that some of the comments and examples of work shown in the previous section will again be seen in this section. This cannot be avoided though, since it is necessary for both sections to show examples of work, and to simply refer to the previous sections does the reader a disservice.

\section{FUNCTIONS}

There were several questions asked pertaining to functions. The primary goal of these questions was to discover what the student felt a function is and how they go about determining functions from graphs. The question that gathered the most information about the students' views on functions was question Q10 from the first interview.

\section{Function Recognition/Definition}

The students were asked three questions to ascertain their views on what a function is and how they recognize functions: Question Q10 from the first interview, and questions F1 and F2 from the followup. We shall begin with questions Q10 and F1.

Q10) I'm going to show you 9 graphs, labeled G1-G9 (see pages 14-16). Please sort the graphs into two piles: If the graph could be the graph of a function, place it in one pile; if not, place it in a separate pile. Explain your criteria for deciding whether or not a graph is a function. What is a function? 


\section{F1) Please clarify your criteria for determining functions.}

Question Q10 has three parts to it: The first part asks the students to identify which of the nine graphs could represent functions; the second question asks them to explain a valid criterion for determining functions; and third part simply asks the students to define functions. If the subject misidentified no more than two graphs, that was considered an acceptable response to the first part. Their criteria had to be valid to be considered an acceptable response to the second part. For the third part, they had to give a reasonable definition of function, such as for every $x$ there can be only one $y$, to be considered a good response. Question F1 was needed since it was discovered during the analysis of the first test that some of the criteria given during the first test were not explained or poorly worded.

In the traditional group, three students had perfect scores identifying the graphs, and two others identified the graphs satisfactorily. Only one student, Tammy, misidentified more than two graphs. Tammy thought that G4, G5, G6, and G8 were not functions. Also, both Tammy and Tuong had invalid criteria for determining functions. They both said that a graph must pass the vertical line test and it must be continuous in order for it to represent a function. On the surface, this may appear odd, since Tuong identified some of the discontinuous functions correctly, but his definition of continuity is that the domain is a continuous interval.

In the Harvard group there was only one perfect score, but only two students, Herb and Hu-Anh, incorrectly identified more than two graphs. Herb missed five graphs, thinking that G3 could represent a function, and also believing that G4, G5, G6, and $G 8$ could not represent functions. Hu-Anh missed six graphs, thinking that $G 1, G 3$, and $\mathrm{G7}$ were all functions, while also identifying G4, G5, and G8 as non-functions.

Not surprisingly, Herb and Hu-anh were also the only Harvard students who had invalid criteria for determining functions. Hu-Anh's criteria were if the graph had no gaps and " $y$ depends on $x$, then it is a function." In the followup she did say that there are functions that are not continuous, such as step functions, but Hu-Anh would still fall back on continuity if the function looked unfamiliar, such as saying G4 is not a function because it is "just points." Also, the graphs of piecewise functions bothered her. For instance, she said that G8 was not a function because it looks like "there's two different equations."

Herb's criteria for determining functions changed from the first interview to the followup. On the first interview, Herb said he identified graphs as functions if they 
looked familiar and if he remembered them from class. He did no better during the followup, stating that a graph represented a function "if it was smooth [and] had no gaps."

The definitions from both groups of students were, for the most part, very informal, but most of them were able to give a satisfactory answer. From the traditional class, three of the students defined function as having one output for every input, such as Tammy's response: "you put in one number and you only get one number out." Ted gave an exceptional definition, saying that with a function, "for every value in the domain there's one unique value in the range." Only Tanya and Tylinn did not give a reasonable definition; Tanya said she could not define function, and Tylinn thought a function was any equation with variables in it.

The Harvard group's most common response was the same as the traditional group's most common response; that is, four of the six Harvard students defined a function as having one value for every input. The remaining two students, Henry and Hu-Anh, did not give a satisfactory definition. Hu-Anh did not state a definition, and Henry said that a function is "a line or a curve."

F2) Originally, you said that were not functions. How would you change these graphs in order for them to represent functions?

Question F2 was asked primarily for two reasons: to provide further insight into what they thought the graph of a function should look like, and to see if the subjects would correct any mistakes they may have made in answering the first question. As long as they made appropriate corrections, it was deemed a satisfactory answer.

Question F2 was useful in that it helped to confirm what their criteria were. For example, when Tuong described how he would change G4 (a graph he incorrectly identified as a nonfunction) into a function, he said he would connect the points to make it continuous. To make G6 a function, Tammy suggested connecting each of the steps "to make it continuous." Herb and Hu-Anh had similar ideas, with both saying they would connect the points of G4 to make it a function; for G2 Herb said he would straighten or smooth out the sharp point. The remaining students offered appropriate changes to the graphs of the nonfunctions, and some of the students, such as Tylinn and Henry, caught a couple of mistakes they made during the initial interview. 


\section{Sketch a Function}

F3a) Sketch a function $f$ such that $f(2)=3$ and $f(1)>f(5)$.

This question was asked to see if students could follow simple directions on graphing a function. If they were able to sketch a function that satisfied the criteria, that was considered satisfactory. Note that the sample sketches included in this section are accurate reproductions of the students' work. The $x-y$ axes are there for the reader's benefit -- on the original work, the axis was usually hand drawn by the student, or the student used the graph paper provided.

Everyone from both groups was able to complete this task satisfactorily. From the traditional group, both Ted and Tanya skeiched what appeared to be an upside down parabola (see Tanya's sketch below), and Tammy and Tuong sketched similar straight lines with a negative slope through the point $(2,3)$ (Tammy's sketch appears below also). Tylinn and Tracy both drew curves for question F3 (see Tylinn's sketch below, and Tracy's sketch on page 67).

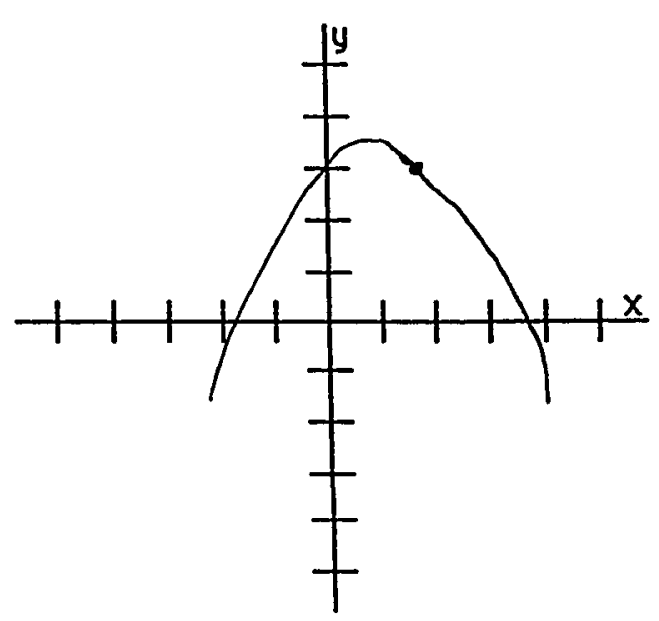

Tanya's Sketch for Problem F3

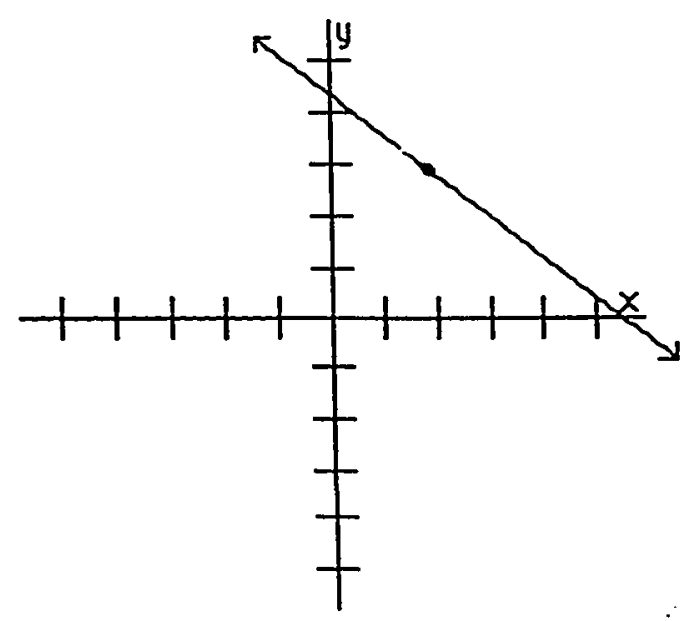

Tammy's Sketch for Problem F3 


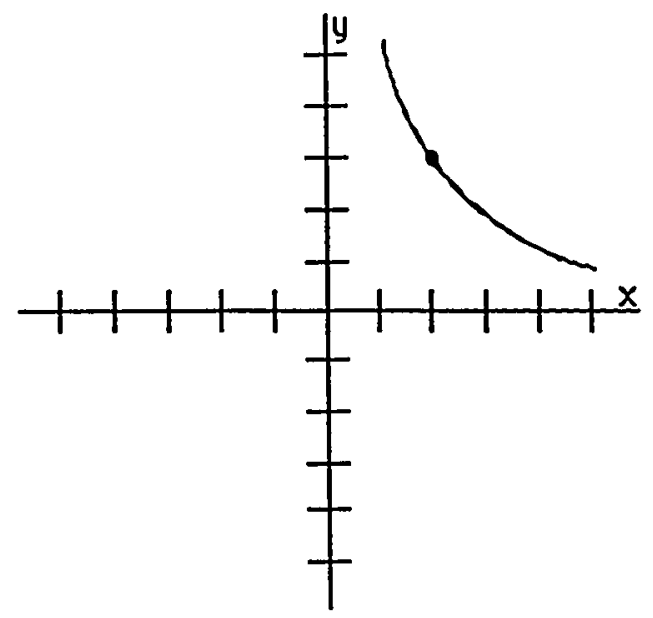

Tylinn's Sketch for Problem F3

The Harvard group was equally successful for this problem. Interestingly, though, there was much more conformity in their responses. In fact, five of the Harvard subjects simply drew a straight line with a negative slope through the point $(2,3)$. Harriet had the only unique response among the Harvard students (see her sketch on page 66).

\section{Interpreting the Graph of a Function}

T2) Suppose the graph below is the graph of $y=f(x)$. By examining the graph, find the following (if there is not enough information on the graph, simply write $n / a$ in the space provided):

a) The precise intervals where $f(x)$ is increasing

b) The $x$ and $y$ intercepts of $f(x)$.

$x$-int

$y$-int

c) Local minimum (approximate)

d) Local maximum (approximate) 


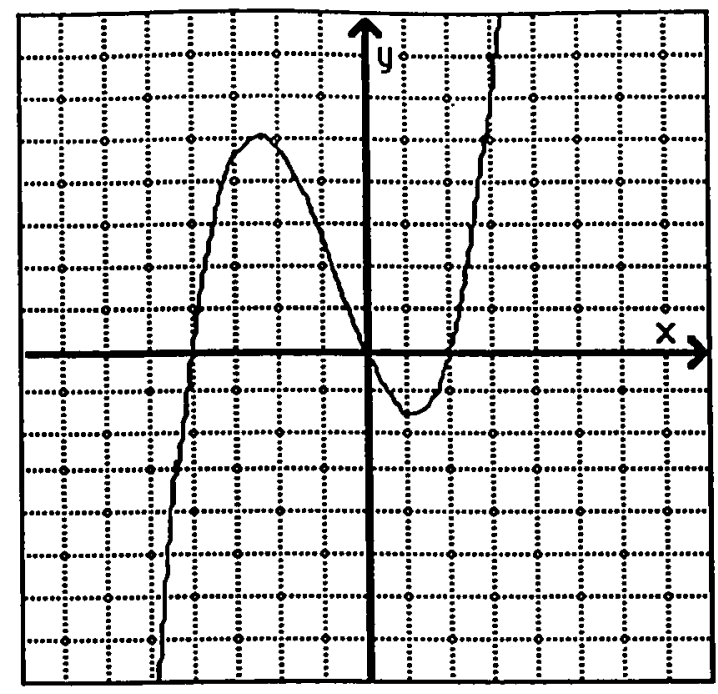

Question T2 was used mainly as a setup for question T3, since that was also about interpreting a graph, only for that question the graph was the graph of the derivative function. This question was also supposed to be a confidence booster, since it should be relatively easy for any calculus student. To be considered an acceptable answer, each question should have the correct answer, with some leeway given for estimation of points on the graph.

Virtually everyone from both groups answered question T2 reasonably well. Some of the notation was nonstandard, but it was all done satisfactorily.

\section{SUMMARYOFRESPONSESONFUNCTIONS}

To give the reader a quick view of how each group did on the questions, below is a summary of the data gathered on functions. Specifically, the summary below tallies the responses to the groups' performance on defining functions, recognizing functions, and their criteria for determining functions. The ability to sketch a function, question F3a, and interpreting the graph of a function, question T2, are not included here, since virtually everyone answered those questions correctly and nothing of any major significance was learned from them. Bold entries indicate a difference of two or more responses between the traditional group and the Harvard group. 
TABLE 2: FUNCTIONS

\begin{tabular}{lcccc}
\hline Category/Question & Responses: & Good & Acceptable & Unacceptable \\
\hline Defining & Traditional: & 4 & 0 & 2 \\
(Question Q10) & Harvard: & 4 & 0 & 2 \\
\hline \multicolumn{1}{l}{ Recognizing } & & & & 2 \\
(Question Q10) & Traditional: & 3 & 2 & 2 \\
\hline Determining & Harvard: & 1 & 3 & 2 \\
(Questions Q10, F1) & Traditional: & 4 & 0 & 2 \\
\hline
\end{tabular}

\section{LIMITS}

With limits, perhaps more than any other topic, the number of correct answers for any particular question is less telling than which specific problems each individual answered correctly. The table at the end of this section should give the reader some insight into the students' understanding of limits, since questions are grouped together based on specific issues about limits. Further analysis of these results will be discussed in Chapter 5, which will give an even better indication of what the students understand about limits.

\section{General}

F13) If for some function $g$, the limit as $x$ goes to 3 is 5 , could we conclude that $g(3)=5$ ? Explain your answer.

This question was asked to see how the student viewed the relationship between the value of the function at a point and the limit at that point. The only proper response is to say no.

From the traditional group, four subjects responded accurately; only Tanya and Tammy answered incorrectly. In the Harvard group, three answered correctly, and Helen, Hannah, and Harriet answered incorrectly. 


\section{Solving Graphically}

T1) For the following questions, find the limit if it exists. If it does not exist, write $\mathrm{n} / \mathrm{a}$ in the space provided. If you're not sure, write NOT SURE. As stated earlier, the small boxes on the graphs represent 1-by-1 unit squares (see graphs on pages 1618).
a) In graph $\mathrm{G2}$, the $\lim _{x \rightarrow 3}$ is :
b) In graph G5, the $\lim _{x \rightarrow 2}$ is:
c) In graph $\mathrm{G8}$, the $\lim _{x \rightarrow 2}$ is:

F10) On the first test you were asked to find some limits, if they existed, by examining the graphs of some functions. Please explain each answer.

Question T1 asked the student to find a limit graphically. Each part was treated as a separate question, since each part addresses a different problem. Part a is relatively easy -- it only asks about the limit of a continuous function. Part b asks for the limit of a discontinuous function, where the limit at 2 does not equal the value of the function at 2. Part $\mathrm{c}$ also features a discontinuous function, only the limit does not exist. The only acceptable answers to parts $a, b$, and $c$ are -2.3 (approximately), -1 , and n/a, respectively. Question F10 was needed since most of the answers in the initial test were given without explanation. The two groups performed about equally on these questions.

In the traditional group, everyone answered part a correctly. For part b, only Tracy and Ted answered correctly. Tylinn answered that the limit did not exist because she felt that the left limit did not equal the right limit. The other three students from the traditional group thought the limit in part $b$ was simply 3 (the value of the function at 2). Tuong justified his answer to part $b$ by saying that "as it gets closer to 2 [for part b] the only value that it could take on is 3." On part c, three traditional students answered correctly (Tracy; Ted, and Tylinn). The other three subjects felt that the limit existed and was 3.9 (again, the value of the function).

In the Harvard group, only Hannah missed part a. She thought the limit did not exist because the function comes to a "sharp point [at 3]. I just remember something 
about a sharp point, like it can't be defined or something like that." Clearly Hannah was confusing the existence of a limit with differentiability. On parts $b$ and $c$, the Harvard group was evenly split -- three had the correct answer, and three did not provide an acceptable answer. Two said they did not know the answer to part $b$, and the third unacceptable answer was given by Harriet, who, like Tylinn, thought the limit did not exist because she felt the left-sided and right-sided limits were not equal. The three students who missed part $c$ all stated they did not know the answer.

\section{Solving Algebraically/Procedurally}

F11) I'm going to give you the following limit questions for you to answer in writing. After you have answered them, please explain each answer (the subject is then handed a sheet with the limit problems seen below).

Find the following limits (if they exist). If the limit does not exist, write n/a in the space provided. You will be asked to explain your answers.

a) $\lim _{x \rightarrow 2} f(x)$, where $f(x)= \begin{cases}3 x+5 & x<2 \\ 3 x+5 & x \succ 2\end{cases}$

b) $\lim _{x \rightarrow 3} f(x)$, where $f(x)=\frac{3 x^{2}-9 x}{x-3}$

c) $\lim _{x \rightarrow-\infty} f(x)$, where $f(x)=\frac{3 x^{2}+2 x+1}{x^{2}-5}$

d) $\lim _{x \rightarrow 4} f(x)$, where $f(x)= \begin{cases}3 x-5 & x \leq 4 \\ 2 x+3 & x>4\end{cases}$

e) $\lim _{x \rightarrow 4} f(x)$, where $f(x)= \begin{cases}2 x+3 & x \prec 4 \\ 5 & x=4 \\ x^{2}-5 & x \succ 4\end{cases}$

Question F11 has several parts to it, and like question T1, each part will be treated separately. Parts $a$ and $b$ are similar, in that they both are asking for the limit at a point which is not in the domain of the function. The answers are 11 and 9, respectively. Part $c$ is strictly procedural, and forces the student to recall some of the 
methods they learned for finding limits -- the answer is 3 . For part $d$ the limit does not exist, since the left-sided limit at 4 does not equal the right-sided limit at 4 , and for part $e, f(4)=5$, but the limit of $f$ as $x$ goes to 4 is 11 .

In the traditional group, three people had the correct answer to part a, while two said the limit did not exist, and one student, Tanya, could not answer the question since, as she put it, "I remember what piecewise functions are but I don't remember how to do them." (This affected Tanya on parts $d$ and e also.) For part $b$, four traditional students had the correct answer, while one student said the limit did not exist since you cannot divide by 0 , and another just made a procedural error. Every traditional student answered part c correctly. On part $d$, four answered correctly, one answered that the limit is 7 (since $f(4)=7$ ) and Tanya could not answer the question. Finally, for part e, three traditional students answered correctly, while two students answered that the limit is 5 (since $f(4)=5$ ); again, because she could not work with piecewise functions, Tanya could not answer the question.

The Harvard group did not do very well on question F11. Hannah could not answer a single question, saying she did not know limits very well. Therefore, the numbers below will be from a total of five Harvard students. With that said, from the remaining five Harvard students, three answered part a correctly, while the other two thought the limit did not exist since the function was undefined at 2 . For part $b$, only two subjects answered it correctly -- the wrong answers include Harriet saying there was no limit since the function is undefined at 3; Herb answering " 0 because it's 0 divided by 0 ;" and Helen answering that the limit is infinity "because there's no value at $x=3$, there's an asymptote. . . and I think the function from both sides goes off to infinity." For part c, three answered correctly. The two wrong answers for part c were Hu-Anh's response that "infinity divided by infinity is 1 " and Helen's answer that the limit is infinity. The question the Harvard group did best on was part $d$, with all five of the remaining students answering it correctly. For part e, only Henry answered correctly. Of the other four, two thought that since the function approaches 11 as $x$ approaches 4 but $f(4)=5$, the limit must not exist; the remaining two thought that since $f(4)=5$, the limit must be 5 . 


\section{Solving Numerically}

F12) A student was given a function $f$ and asked to find the limit as $x$ approaches 0 . He plugged in numbers on each side of $O$ and made the following table:

$\begin{array}{rl}x & f(x) \\ -.1 & 0.9 \\ -.01 & 0.99 \\ -.001 & 0.999 \\ -.0001 & 0.9999 \\ -.00001 & 0.99999 \\ -.000001 & 0.999999 \\ -.0000001 & 0.9999999 \\ .1 & 1.1 \\ .01 & 1.01 \\ .001 & 1.001 \\ .0001 & 1.0001 \\ .00001 & 1.00001 \\ .000001 & 1.000001 \\ .0000001 & 1.0000001\end{array}$

a) What can you conclude about the limit of the function $f$ as $x$ approaches 0 ?

b) Would your answer change if you were told that $f(0)=7$ ?

c) What could you say about the limit if 0 is not in the domain of $f$, that is, $f(0)$ is undefined?

Part a of question F12 is fairly straight forward; the only answer is 1 or a reasonable explanation as to why the limit may not be 1 . Parts $b$ and $c$ explored the subject's views of the relationship between the value of the function at a point and the limit as $x$ approaches that point. Students' answers to parts $b$ and $c$ should be the same as their answer to part a. 
In the traditional group, there were five students who were asked question F12 (unfortunately, Tanya, as well as Hannah from the Harvard group, was not asked this question. She and Hannah were the first two subjects to take the followup test, and this question was added after the fact.). On part $a$, the five traditional students answered 1, and on part $b$ three of the five said they would not change their answer (the other two thought that since $f(0)=7$, the limit at 0 must also be 7 ). On part $c$, four students answered correctly, with only one student, Tylinn, thinking that the limit would not exist.

In the Harvard group, of the five students asked this question, all five answered part a correctly (they all said the limit would be 1 ). On part b, only two answered correctly, with two saying the limit would not exist and one saying that she did not know. On part c, there was a minor improvement, with three answering correctly and two saying the limit would not exist.

\section{Summary of Responses from Limits}

The questions asked on limits addressed some of the important ideas associated with limits. The subjects' responses to questions on the same topic have been grouped accordingly in Table 3, below. Note that for the purpose of grouping the students' responses to similar questions, part a of question F12 was treated as if it was asking about a continuous function (recall that it could be answered as if it were not continuous at 1 , but no one responded that way). Also, question F12 was only asked of five students from each group, so the responses from those questions will add up to 5 .

The table begins with question F13, which asks the subjects whether or not the value of the limit at a point tells you the value of the function at that point. Some of the other questions also deal with that issue, however only question F13 begins with the assumption that the limit exists; the other questions are asking whether or not the limit exists. Questions T1a and F12a are grouped together, since they both ask the student to find the limit of a continuous function. Questions F11a, F11b, and F12c are all questions dealing with limits at an undefined point. Questions T1b, F11e, and F12b are all questions in which the value of the limit at a point is not equal to the value of the function at that point. Question T1C and F11d are questions on discontinuous functions in which the limit in question does not exist. Question F11c is the only question asked on the limit at infinity. The answers for questions that are grouped together appear in the same order as they do above. Bold entries indicate a difference of two or more responses 
between the traditional group and the Harvard group.

TABLE 3: LIMITS

\begin{tabular}{|c|c|c|c|}
\hline Category/Question & Responses: & Good & Unacceptable \\
\hline Value of Limit, Function & Traditional: & 4 & 2 \\
\hline (Question F13) & Harvard: & 3 & 3 \\
\hline Continuous Function & Traditional: & 6,5 & 0,0 \\
\hline (Questions T1a, F12a) & Harvard: & 5,5 & 1,0 \\
\hline Limit, Undefined Point & Traditional: & $3,4,4$ & $3,2,1$ \\
\hline (Questions F11a, F11b, F12c) & Harvard: & $3,2,3$ & $3,4,2$ \\
\hline Limit $\neq$ Value of Function & Traditional: & $2,3,3$ & $4,3,2$ \\
\hline (Questions T1b, F11e, F12b) & Harvard: & $3,1,2$ & $3,5,3$ \\
\hline Discontinuous, no limit & Traditional: & 3,4 & 3,2 \\
\hline (Questions T1c, F11d) & Harvard: & 3,5 & 3,1 \\
\hline Limit at Infinity & Traditional: & 6 & $\mathbf{0}$ \\
\hline (Question F11c) & Harvard: & 3 & 3 \\
\hline
\end{tabular}




\section{CONTINUITY}

There were only a few questions about continuity. The questions were primarily about defining and recognizing continuity. For further insights into the students' thinking on continuity, see the subsection on differentiability later in this chapter.

F4) Can you tell me what it means for a function to be continuous?

As stated earlier, formal definitions were not expected to be given by the students. If the subject said that a continuous function is a function that can be graphed without any holes or gaps, that was considered acceptable.

In the traditional group, only two students, Ted and Tracy, gave an acceptable definition, saying that the graph of a continuous function can have no breaks or gaps. There were various incorrect definitions, such as Tylinn's -- "It's like one graph can depict every situation." Tammy said a function is continuous if "at no point is it undefined," and Tanya felt that for a function to be continuous "it has to pass both the vertical and horizontal line test for it to be continuous, I think." Tuong's response was similar to Tammy's, saying that a function is continuous as long as the domain is a continuous interval.

In the Harvard group, Henry had a very good definition, saying that a continuous function should have no breaks and that "the limit coming from the left and right will be the same number." Four other Harvard students had acceptable responses, saying that the graph should have no breaks or gaps. Only Hu-Anh did not verbalize an acceptable definition for continuity. She said that she was not sure how you would define it.

F5a) I'm going to hand you a stack of cards labeled i, ii, iii, and iv (see below). On each of these cards there is a piecewise function. Identify which of the four piecewise functions are continuous.
i) $f(x)= \begin{cases}x+3 & x \leq 4 \\ 4 x-9 & x>4\end{cases}$
ii) $f(x)= \begin{cases}2 x-3 & x \leq 5 \\ 2 x+3 & x>5\end{cases}$
iii) $f(x)= \begin{cases}\frac{1}{5} x^{2}+x-7 & x \leq 5 \\ x-2 & x>5\end{cases}$
iv) $f(x)= \begin{cases}\frac{1}{2} x^{2}-x-7 & x \leq 4 \\ 3 x-15 & x>4\end{cases}$ 
F6a) Here are the graphs of those same four functions (see pages 24 and 25). Now that you see the graphs, which ones do you consider continuous?

Questions $\mathrm{F} 5 \mathrm{a}$ and $\mathrm{F} 6 \mathrm{a}$ both examine continuity, only from different points-ofview; F5 approaches the problems analytically, and F6 takes the graphical approach. For question F5, part a, the student must use an appropriate method for determining continuity for it to be considered a good answer. The methods the students usually used were checking the value of the transition points for both pieces of the piecewise function, or sketching a graph of the function. To be considered a good answer for question F6, part a, the student had to recognize that functions $i$, iii, and iv are continuous; anything else was unacceptable.

Only three subjects from the traditional group answered question F5, part a, correctly. Of the three, two subjects simply checked the value at the transition point for both pieces of the function, and one student chose to graph the functions. The other three traditional subjects missed this question for different reasons. Tylinn thought none of the piecewise functions were continuous because each one was defined by more than one equation, while Tuong thought they were all continuous since each of the functions had a domain of all real numbers. Tanya basically had no idea how to check for continuity, and her idea of continuity is that the function must pass the horizontal line test.

Five of the Harvard students answered question F5a correctly. Four of them checked the value at the transition point for each piece of the function, while the fifth one (Herb) used his graphing calculator to graph the functions. Only Harriet did not answer this problem acceptably. She basically guessed (incorrectly) that function ii was continuous, saying "the function is just going up; it just goes up faster here [at $2 x+3$ ]. It looks continuous to me." For the other three functions, her approach was better, but still not satisfactory. What Harriet did for functions $\mathrm{i}$, iii, and iv was to substitute the value of the transition point into one piece of the function and a number close to the transition point into the other piece of the function. For example, for function $i$ she substituted 4 into the first piece of the function and 4.1 into the second piece and since those two values were close she concluded that function $i$ is continuous. This method has some serious conceptual problems, since if the two pieces were close but not continuous (at the transition point) she would judge the function to be continuous.

The Harvard group again did better than the traditional group when the functions were presented graphically. In the traditional group, four of the students recognized the 
continuous functions graphically. Both Tuong and Tanya did not recognize the continuous function, and both were fairly confused on continuity. Tanya thought that a graph should pass the horizontal line test to be continuous, therefore she felt that only functions $i$ and ii are continuous. Tuong felt that as long as the domain of the function was a continuous interval, the function was continuous. Therefore, he thought all four functions were continuous. Everyone from the Harvard group recognized continuous functions when presented graphically.

\section{Continuity and Differentiability}

The two questions below are the only two questions that ask (directly) about the relationship between continuity and differentiability. However, question T5 as well as part $b$ to question F5 indirectly approach the topic (see later in this chapter).

F7a) If a function is continuous at $x=2$, must it be differentiable at 2 (that is, must the derivative exist at 2)? Sketch an example or counterexample.

F7b) If a function is differentiable at $x=5$ (that is, the derivative exists at 5), must it be continuous at 5 ? Sketch an example or counterexample.

Questions F7a and F7b were to-the-point questions to see if they knew how differentiability and continuity are related. One must answer both questions correctly (the answer to F7a is no, the answer to F7b is yes) for it to be considered a good answer. If they answered one or both questions incorrectly, that showed that they did not fully understand how the two ideas are related, and therefore that was considered unacceptable. The sketch of an example or counterexample was not stressed, and most people simply referred to the graphs of functions $i$, ii, iii, and iv, from problem F6, to support their claim.

Only two traditional subjects understood that differentiability implies continuity and that continuity does not imply differentiability. Two of the traditional students with wrong answers answered yes to both parts of F7. Tanya, who also answered incorrectly, thought the answer was no to both parts. Tanya cited the graph for function iv as a counterexample to part $a$, thinking graph iv represented a continuous nondifferentiable function. She also used the graph of function iii as a counterexample to part b. Tylinn answered yes to part $a$, and used the graph of function $i$ to support her belief. She then 
answered no to part $b$, referring to the graph of function ii as being differentiable but not continuous.

Four of the Harvard subjects answered question F7 correctly. Herb and Hannah answered incorrectly, with Herb answering no to both parts of the question. Hannah answered yes to part $a$, and answered no to part $b$, using the graph of function ii to support her claim that a function could be differentiable but not continuous.

\section{Summary of Results from Continuity}

The Harvard group did much better than the traditional group answering questions on continuity. Table 4, below, summarizes the groups' responses on defining continuity, recognizing continuity (both analytically and graphically, respectively), and understanding the relationship between continuity and differentiability. Bold entries indicate a difference of two or more responses between the traditional group and the Harvard group.

TABLE 4: CONTINUITY

\begin{tabular}{lcccc}
\hline Category/Question & Responses: & Good & Acceptable & Unacceptable \\
\hline Define & Traditional: & 0 & 2 & 4 \\
(Question F4) & Harvard: & 1 & 4 & 1 \\
\hline Recognize & Traditional: & 3,4 & 0,0 & 3,2 \\
(Questions F5a, F6a) & Harvard: & 5,6 & 0,0 & 1,0 \\
\hline Continuity and Differentiability & Traditional: & 2 & 0 & 4 \\
(Question F7) & Harvard: & 4 & 0 & 2 \\
\hline
\end{tabular}




\section{DIFFERENTIATION}

Differentiation was the most explored topic of all. The students were asked to define derivative, estimate derivatives, differentiate functions, interpret the graph of a derivative, and more. This topic showed the largest difference in the two groups.

\section{General}

Q8) Can you tell me what a derivative is?

Question Q8 was the first real calculus question in the interview. A good response was to say that a derivative is the slope of the tangent line at a point. Other acceptable responses were to say that the derivative is the slope of the curve or slope of the function, or calling the derivative a rate of change.

In the traditional calculus group, two subjects had good responses, saying that the derivative is the slope of the tangent line; another two had acceptable responses -- one said rate of change and the other said the derivative "tells you where the graph is going, whether it is increasing or decreasing." The remaining two subjects, Tanya and Tuong, both initially tried to answer by explaining the process of differentiating. For example, Tanya said that for a polynomial, "you take the power and you put it in front." She did, however, allude to a connection between the derivative and a minimum point -- "like when you're graphing, [the] first derivative is equal to a minimum point of a . . . parabola or something." Tuong conceded that he did not really know what a derivative is, and concluded that "with math understanding it and being able to explain it is different from actually doing it." To summarize, the traditional group had four responses that were at least acceptable.

For the Harvard group, virtually every student used the word slope, although one of them still did not give an acceptable definition. Two said the derivative is the slope of the tangent line, one said it is both the rate of change and the slope of the "equation," two others said it was the slope of the curve, and only Herb could not give a reasonable definition. Herb answered in more of a procedural manner, saying "I can do one for you." He then proceeded to write on his paper that the derivative of $x^{2}$ is $2 x$. When asked how $2 x$ relates to the first function, he replied "I think it means the slope." Question: The slope of what? "I can't remember." Herb's answer was close to being considered acceptable, but since he did not know how $2 x$ related to his original function, 
it was considered an unacceptable response. Therefore, five of the Harvard students knew that the derivative is related to slope and were able to give sufficient responses to the question.

\section{Q9) Can you explain the relationship between a derivative and a limit?}

Question Q9 was asked to see if the subjects could tie together two important concepts from the first semester of calculus. It turned out to be too difficult for them, perhaps because the only way to answer this correctly is to paraphrase the formal definition of derivative. For instance, to answer this correctly, the subject would have to talk about the limit of secant lines, or the limit of the difference ratio as the denominator goes to zero. Both ideas are fairly sophisticated. No one from either the Harvard or traditional group gave an acceptable answer, although a couple were on the right track.

The wrong answers from both groups ranged from close attempts to the absurd. In the traditional group, Ted offered perhaps the best attempt, saying that "the derivative of a function is the limit, with say. . . respect to $x$. . . is the limit as $x$ approaches 0 of that function." He then wrote on scratch paper "dx/dt $=\lim$ as $t \rightarrow 0 \quad \Delta x / \Delta t . "$ The most common wrong answer given for this question involved describing the limit and the derivative, but not necessarily linking the two, such as Tylinn's response: "A limit is when the number is approaching a certain point, and a derivative tells you where the graph is going, whether it is increasing or decreasing." Tanya probably remembered something of the formal definition, but her translation was very deficient, saying that "I know [the derivative] is like the limit as $\mathrm{x}$ approaches infinity of the function."

In the Harvard group no one even came close to answering this correctly. Two of them just said that they could not answer the question. Herb and Hu-Anh were probably the most confused though, since they both essentially answered that the limit and the derivative are the same thing. Herb asked "aren't you supposed to get the same answer [as the derivative] when you take the limit?" Hu-Anh almost echoed those words, saying "A limit is a derivative, I don't know." She then followed that by saying "a limit narrows things down into smaller pieces." Helen's response was accurate, saying that "a limit is what the function goes to. . . the derivative can be another function," but like some students did from the traditional group, she did not link the two concepts together.

In conclusion, no one gave an acceptable answer to this question. Ted gave the best response of all the subjects combined, and the traditional group's responses were 
somewhat clearer than the Harvard group's responses.

\section{. Estimating the Derivative}

There were three questions that fell into this category: Question Q11 from the first test, and questions F8a and F3b from the followup test.

Q11) The table below is a partial table of values for some unknown function $f$. Assuming the function is differentiable at 3 , is it possible, with the given information, to approximate $f^{\prime}(3)$ ? If so, then approximate $f^{\prime}(3)$ and explain how you did so; if not, explain why not.

\begin{tabular}{|c|c|c|c|c|c|c|c|c|}
\hline$x$ & 2.8 & 2.9 & 3.0 & 3.1 & 3.2 & 3.3 & 3.4 & 3.5 \\
\hline$f(x)$ & -1.325 & -2.291 & -3.300 & -4.351 & -5.443 & -6.576 & -7.750 & -8.963 \\
\hline
\end{tabular}

F8a) Without actually finding the derivative, that is, without using any techniques of differentiation, how would you estimate $f^{\prime}(2)$, where $f(x)=x^{x}$ ? You need not actually attempt to estimate the derivative, just explain the procedure.

The two questions above involve estimating the derivative without knowing the actual derivative function. As the reader can see, these questions are posed with different representations, one being numerical and the other algebraic. The function $f(x)=x^{x}$ was chosen since it was believed that very few students would be able to differentiate it quickly, if at all. When judging their responses, the student's approach was given the most weight. The best response was to use the difference quotient correctly, while graphing the function and estimating the slope of the tangent was considered acceptable.

On the numerical problem (question Q11), only Tracy and Ted from the traditional group used the difference quotient (correctly) to estimate the derivative. A third subject, Tammy, used the difference quotient, but picked her points poorly. Tammy picked the points $(2.8,-1.325)$ and $(3.5,-8.963)$, the endpoints of the table. She also made an arithmetic error. but her method was appropriate, therefore her answer was considered acceptable. Tanya felt that it was possible to estimate the derivative using a table of values, "but I don't remember how." Both Tylinn and Tuong 
felt that it was not possible to estimate the derivative, but for different reasons. Tuong felt that a table simply does not give you enough information to estimate a derivative. Tylinn was the most confused of the group, saying that the estimation was not possible because "the approximation is not reaching any particular point. The function is going downward, to negative infinity. If the function is going to negative infinity. . . there shouldn't be any derivative you can derive it from. . . the function."

Five of the Harvard students gave at least an acceptable answer to the numerical problem. Two of the subjects, Helen and Hannah, plotted the points and guessed the slope, although their answers were crude (Helen guessed -3 , Hannah guessed -1 ), while three of them used the difference quotient. Only one Harvard student, Herb, could not give an acceptable answer.' In fact, Herb did not even understand the word differentiable at first. After it was explained to him he said that a table of values "isn't enough information [to find a derivative]. I couldn't find a formula [that fit the given values]."

For the algebraic problem, Tracy and Ted again suggested using the difference quotient, although not using those exact words. For example, Tracy said she would take a "couple of values of $f(x)$ and just finding the rise over run. You'd want to use values very close to 2." Tylinn also suggested using the difference quotient, and she wrote that the derivative is equal to $(f(2+h)-f(2)) / h$. However, it seemed that she may have simply been parroting that definition, since when asked for examples of $h$ she could not offer any, saying only that $h$ represents "the distance from $x$." Therefore, her answer was only considered acceptable. Of the remaining three traditional subjects, Tammy said that the derivative is the "slope of the tangent" but did not say how she would find that; therefore her answer was considered unacceptable. The other two traditional subjects did not know how to estimate the derivative.

As they did for the numerical question, five Harvard subjects gave at least an acceptable answer to question F8a. Helen, as did two other Harvard students, said she would use the difference quotient. "l'd use the difference quotient to estimate the derivative," Helen said. "[For h] l'd use .01. (f(2.01) - $f(2)) / .01$. You can take smaller and smaller values of $h$ [to estimate the derivative]." Two other Harvard students suggested sketching the function and the tangent line at 2 and then estimating the slope of the tangent line. Only Hu-Anh did not satisfactorily answer the question. HuAnh sketched a rough graph of $f$, then sketched the tangent at $(2,4)$. However, instead of finding the slope of the tangent line, she proceeded to find the equation of the tangent line and then substituted 2 into that equation to estimate the derivative. 
F3b) Sketch a function $f$ such that $f(2)=3$ and $f(1)>f(5)$. Can you tell me what the derivative of the function you have sketched would look like?

Question F3b was asked to see if the student could look at a sketch he/she created and then approximate the graph of the derivative. Responses to this question were indeed dichotomous; any reasonable approximation of the derivative was considered a good answer, and anything else was unacceptable. As noted for part a of question F3, sample sketches below are exact reproductions of the students' work. The $x-y$ axes are included for ease of reading, and are not part of the student's sketch.

For question F3b, we again see that the Harvard group did better on the topic of derivatives. Everyone in the Harvard group who was asked this question answered it satisfactorily (Hannah, unfortunately, was not asked this question). For example, HuAnh said the derivative would be a constant horizontal line around $y=-2$ (see her sketch below), and Harriet sketched the derivative of her function (see below).

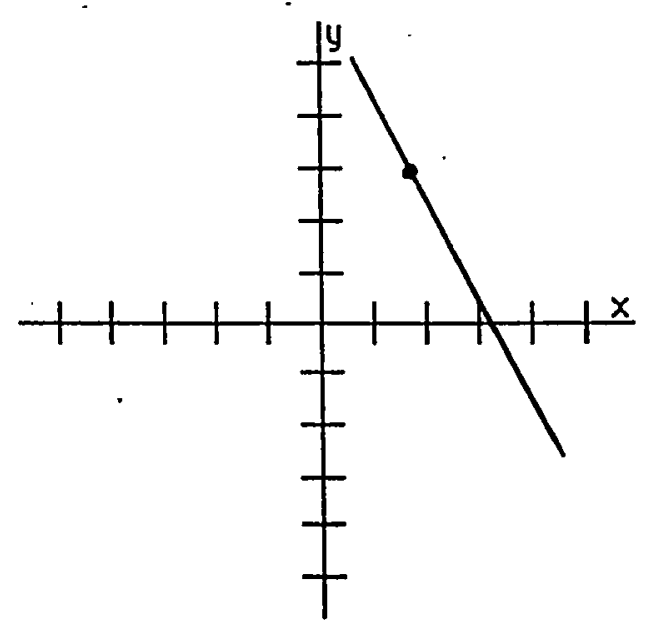

Hu-Anh's Sketch for Problem F3

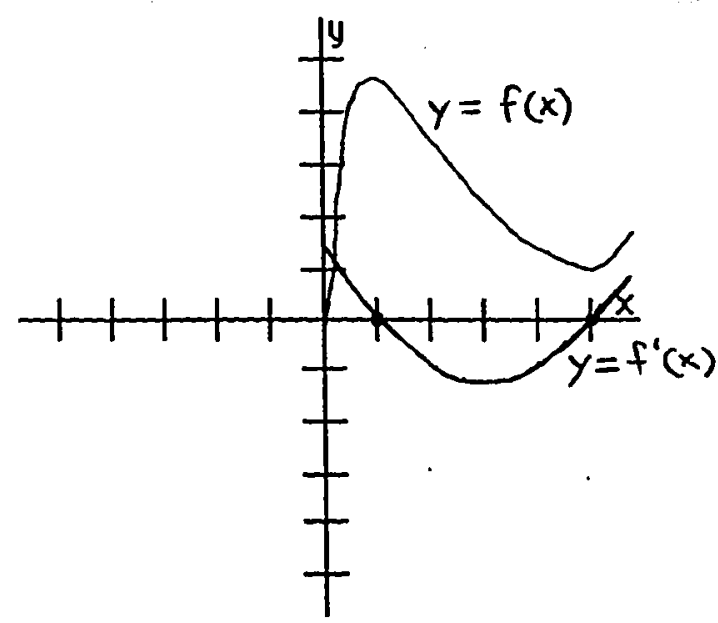

Harriet's Sketch for Problem F3

In the traditional group, of the five students asked question F3b, only Tuong, Tylinn, and Ted answered it satisfactorily (Tanya was not asked this question). For Tuong to answer the question, he needed to estimate the equation of the line he drew first, and then took the derivative of that function (on several occasions Tuong displayed this preference for working with an analytic representation). Ted was very specific in his answer, saying that the derivative of the function he drew (see below) would be positive in the interval from 1 to 2,0 at 2 , and negative from 2 to 5 . The two traditional 
students who could not satisfactorily answer the question were Tracy and Tammy. Tracy answered that the derivative "would be a continuously decreasing function" since her original function was decreasing. As one can see by her sketch of the function for part a of the question (see below), that is not the case.

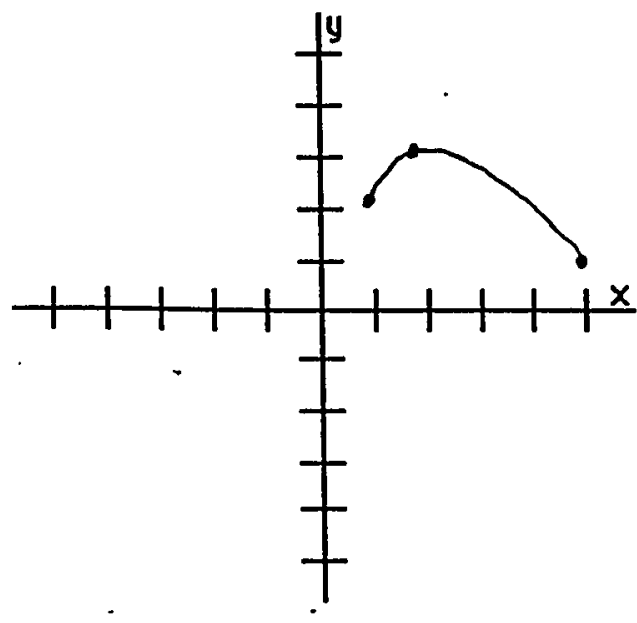

Ted's Sketch for Problem F3

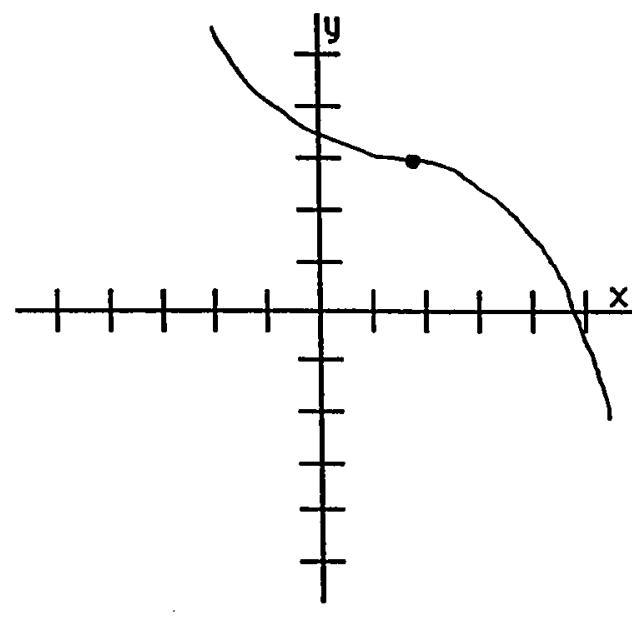

Tracy's Sketch for Problem F3

Tammy, who for the first part of the question drew a straight line with a negative slope going through $(2,3)$, was confused on how a straight line could have a tangent. When asked what the derivative would look like, she said "well, there's nothing that would be tangent to it. I don't know, I don't think there's anything, because it's the slope of the tangent but there's no line tangent to this, and the derivative is the slope. It's constant." Question: So it doesn't exist, or it exists and it's constant? "There's nothing tangent to this so I don't think it can be graphed." Question: So it doesn't exist? "It exists but I don't know how to graph it." Although at one point she did say the derivative is a constant, this clearly is not a satisfactory answer.

\section{Finding the Derivative}

There were three questions that involved finding the derivative: $T 4, F 5 b$, and F15. The two groups performed about equally on these tasks.

T4) On the graph below, the line $L$ is tangent to the graph of $y=f(x)$ at the point $(5,3)$. Find the following: 

a) Find $f(5)$
b) Find $f^{\prime}(5)$

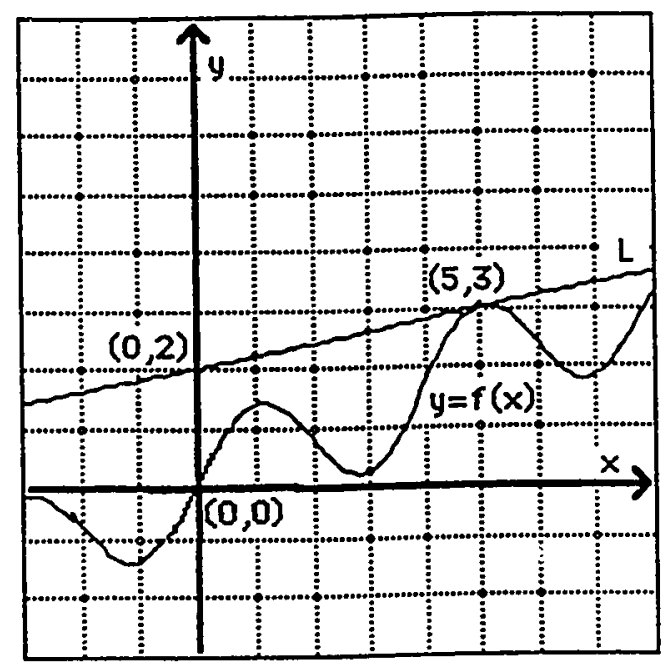

Part a of question T4 was primarily a setup question for part $b$, and the only acceptable answer was 3. Part b was asked to see if the subject understood the relationship between the tangent line and the derivative; the only acceptable answer was to say that $f^{\prime}(5)$ is equal to the slope of line $L$, which is $1 / 5$.

For this question, five of the traditional students answered part a correctly and three of those five answered part $b$ correctly. The two students (Tracy and Tuong) who answered part $a$ but missed part $b$ both had the same wrong answer; they answered 3. However, they came to that answer by different means. Tracy first found the equation of line $L$ and then substituted 5 into that equation, while Tuong simply observed the point $(5,3)$ on the tangent line and assumed 3 was the value of the derivative at 5 . The final traditional student, Tanya, missed both parts of the question. For part $a$, she first found an incorrect equation of line $L$ and took the antiderivative of that to find the original function. Using this approach, she found the original function to be $f(x)=(1 / 10) x^{2}+$ $4 x$. She then substituted 5 into that equation to answer $45 / 2$ for part a. For part $b$, she took the derivative of her function and found $f^{\prime}(5)=5$.

Every Harvard student answered question T4, part a, correctly, and four of them answered part $b$ correctly. Hannah and Hu-Anh were the only Harvard students who missed part b. Hannah's answer was close -- she said that the answer was only approximately $1 / 5$ because $1 / 5$ "is not the slope of the curve, it's only the slope of the line tangent to the curve at that point" -- but she made a conceptual error, therefore her answer was considered unacceptable. Hu-Anh answered 7 on part $b$ by making a couple 
of mistakes; she first found an incorrect equation of line $L$ (she transposed the numbers when calculating the slope), and she then substituted 5 into that equation to arrive at her answer.

F5b) What is the derivative of $f(x)=x^{x}$ ? Feel free to use any of the techniques you learned.

Question F5, part b, was found to be too difficult for most of the students, as was suspected. The only acceptable answer was $f^{\prime}(x)=x^{x}(1+\ln x)$, and to find the answer one must recall some fairly sophisticated rules of differentiation.

Only one subject from the traditional group, Tammy, could differentiate $y=x^{x}$. Tylinn said she did not know how to do it, while three of the traditional students thought the derivative was $x x^{x-1}$. Tuong had the most interesting wrong answer, since he thought the derivative was 1. Question: You mean a constant 1? "I think so because if you take the derivative of this [the exponent] you get 1, and if you take the derivative of this [the base] you get 1 , [and their product is 1] so the derivative is $1 . "$

From the Harvard group, Henry knew to take the logarithm of both sides of $y=x^{x}$, but that was as far as he could go. Three others said they did not know what the derivative was, while two of the Harvard students answered that the derivative is $x x^{x-1}$.

F15) Find the equation of the line tangent to $y=x^{2}$ at the point $(3,9)$.

Question F15, like question T4 above, was asked to see if the students understood how tangent lines and derivatives are related; however, this question explores the issue from an analytical representation. The only good response was the correct answer of $y=6 x-9$. If the student knew that the slope should be 6 but did not know what to do with that, it was considered acceptable since at least they understood that the slope was $f^{\prime}(2)$.

Three students from each group were able to find the equation of the line tangent to $y=x^{2}$ at $(3,9)$. Also, two of the Harvard students and one traditional subject knew that the slope of the tangent line should be 6 but did not successfully complete the problem. 


\section{Differentiability}

There were only three questions that explored differentiability: Questions T5, F5 and F6. We begin with question T5, which explored the students' understanding of differentiability at a point. Questions F5 and F6 were asked to see if the student could recognize differentiable functions.

T5) Let $f(x)=\left\{\begin{array}{ll}a x & x \leq 1 \\ b x^{2}+x+1 & x>1\end{array} \quad\right.$ Find $a$ and $b$ so that $f$ is differentiable at 1 .

Question T5 was asked to see if they understood that for a function to be differentiable at a point, the rate of change must be the same on both sides of that point (that is, the left-sided derivative must equal the right-sided derivative). Of course, to answer this question correctly, one must also understand that differentiability implies continuity. It turned out to be too difficult for the students from both groups, perhaps because of the overly mechanical nature of this question. For instance, to solve this problem you need to solve a system of equations, a topic that many students never fully understand in algebra.

No one from either group made any substantial progress, although some of the comments were interesting. For example, Ted thought the function would be differentiable for any real numbers $a$ and $b$, since the function is a line for any number up to and including 1. He concluded that "b doesn't really play a factor in this." Tylinn took the derivative of both "pieces" at 1 , but was unable to do anything else with this problem. "I forgot how to do this," she said. Everyone else either did not answer or wrote a couple of vague ideas that made little sense.

The Harvard group did not have many interesting comments on this question, simply because every Harvard student except Hu-Anh either left it blank or wrote on their paper "don't know." Hu-Anh took the derivative of both pieces of the function, but like Tylinn, she had no idea what to do after that.

F5b) I'm going to hand you a stack of cards labeled i, ii, iii, and iv (see below). On each of these cards there is a piecewise function. Which ones are differentiable?
i) $f(x)= \begin{cases}x+3 & x \leq 4 \\ 4 x-9 & x>4\end{cases}$
ii) $f(x)= \begin{cases}2 x-3 & x \leq 5 \\ 2 x+3 & x>5\end{cases}$ 

iii) $\quad f(x)= \begin{cases}\frac{1}{5} x^{2}+x-7 & x \leq 5 \\ x-2 & x>5\end{cases}$
iv) $f(x)= \begin{cases}\frac{1}{2} x^{2}-x-7 & x \leq 4 \\ 3 x-15 & x>4\end{cases}$

FEb) Here are the graphs of those same four functions (see pages 24 and 25). Which ones are differentiable?

Parts b of questions F5 and F6 ask the student to identify differentiable functions; F5 approaches the problems analytically, and F6 takes the graphical approach. There are no results worth reporting for question $F 5 b$, since no one from either group was able to determine differentiability when given the functions analytically. (This may have occurred since, as in question $\mathrm{T5}$, the problem requires understanding one-sided derivatives, a topic given little time in either curriculum.) The groups also did poorly on question F6, part $b$, although a few students were able to answer it correctly. Question F6b could only be answered one way; that is, the only good answer was to identify function iv as the only differentiable function. Anything else was considered unacceptable.

Only one traditional student, Tracy, recognized the differentiable function. Ted and Tammy thought that functions $\mathrm{i}$, iii, and iv were differentiable, and another two, Tuong and Tylinn, thought that all four functions were differentiable. Tanya was the most confused, thinking that functions $i$, ii, and iii were differentiable because at the transition points, there's an "obvious change," whereas with iv, the change is smooth.

Only Helen and Harriet knew that function iv was the only differentiable function. Of the other four Harvard students, everyone thought that functions i, iii, and iv were differentiable, except Hannah, who thought that all of the functions were differentiable, since "you can draw the derivative function of these [functions]." She elaborated that the derivative function would have no gaps or holes, so she assumed the derivative existed everywhere for all four functions. 
Interpreting Derivatives

T3) Suppose the graph below is the graph of $y=g^{\prime}(x)$ (that is, it is the graph of the derivative of $\mathrm{g}$ ). By examining the graph, please find the following information for the function $g(x)$ (if there is not enough information on the graph, simply write $\mathrm{r} / \mathrm{a}$ in the space provided):

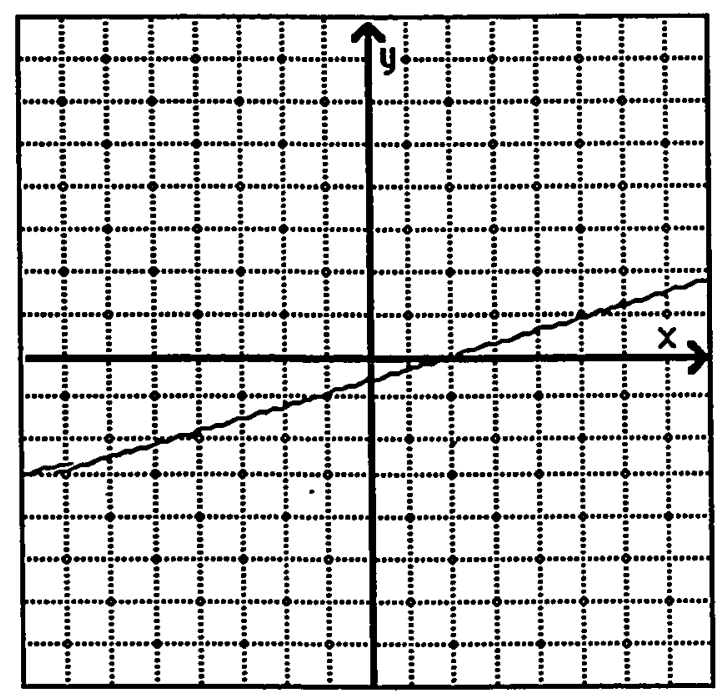

a) The precise intervals where $g(x)$ is increasing

b) Local minimum (approximate)

c) Local maximum (approximate)

This question was designed to see if the subject could gather information about the original function given the graph of its derivative. The answer for part a is the interval $(2, \infty)$, and for part $b$ the answer is $x=2$. Part $c$ has no answer other than "not applicable." The Harvard group seemed to have a clear advantage interpreting the graph of a derivative.

Given the graph of the function $g^{\prime}(x)$, only two traditional subjects could correctly identify where a minimum point would be for the original function $g(x)$ and the intervals in which $g(x)$ is increasing. Also, four of the traditional subjects knew that the graph gave no information about a maximum point. In the Harvard group, four 
subjects found the minimum point of $g(x)$ and five found the correct interval in which $g(x)$ is increasing. Five of the Harvard subjects knew that the graph gave no information about a maximum point.

\section{Summary of Responses from Differentiation}

Table Five, below, summarizes the groups' responses on defining the derivative, estimating derivatives, solving derivatives, recognizing differentiability, and interpreting the graph of a derivative (finding the minimum, maximum, and the intervals in which the function is increasing). Note that there were two questions on recognizing differentiability, questions $\mathrm{F} 5 \mathrm{~b}$ and F6b; however since no one was able to answer F5b correctly, it is not included in the summary below. A look at Table 5 gives a clear picture of the Harvard group's superior performance on almost every question in this section. Bold entries indicate a difference of two or more responses between the traditional group and the Harvard group.

TABLE 5: DIFFERENTIATION

\begin{tabular}{|c|c|c|c|c|}
\hline Category/Question & Responses: & Good & Acceptable & Unacceptable \\
\hline Define & Traditional: & 2 & 2 & 2 \\
\hline (Question Q8) & Harvard: & 2 & 3 & 1 \\
\hline $\begin{array}{l}\text { Estimate } \\
\text { (Questions Q11, F8a, F3b) }\end{array}$ & $\begin{array}{l}\text { Traditional: } \\
\text { Harvard: }\end{array}$ & $\begin{array}{l}2,2,3 \\
3,3,5\end{array}$ & $\begin{array}{l}1,1,0 \\
2,2,0\end{array}$ & $\begin{array}{l}3,3,2 \\
1,1,0\end{array}$ \\
\hline Solve & Traditional: & $3,1,3$ & $0,0,1$ & $3,5,2$ \\
\hline (Questions T4, F5b, F15) & Harvard: & $4,0,3$ & $0,0,2$ & $2,6,1$ \\
\hline Recognize & Traditional: & 1 & 0 & 5 \\
\hline (Question F6b) & Harvard: & 2 & 0 & 4 \\
\hline Interpret & Traditional: & $2,4,2$ & $0,0,0$ & $4,2,4$ \\
\hline (Questions T3a, T3b, T3c) & Harvard: & $4,5,5$ & $0,0,0$ & $2,1,1$ \\
\hline
\end{tabular}




\section{INTEGRATION}

There were only three questions in the study that examined integration, two of which focused on solving an integral. The third question asked whether or not a derivative function is related to one unique function.

\section{Solving Integrals Graphically}

T3d) On the axis below, the line shown is the graph of $g^{\prime}(x)$ (that is, it is the graph of the derivative of $g$ ). Sketch a graph of what the original function might look like.

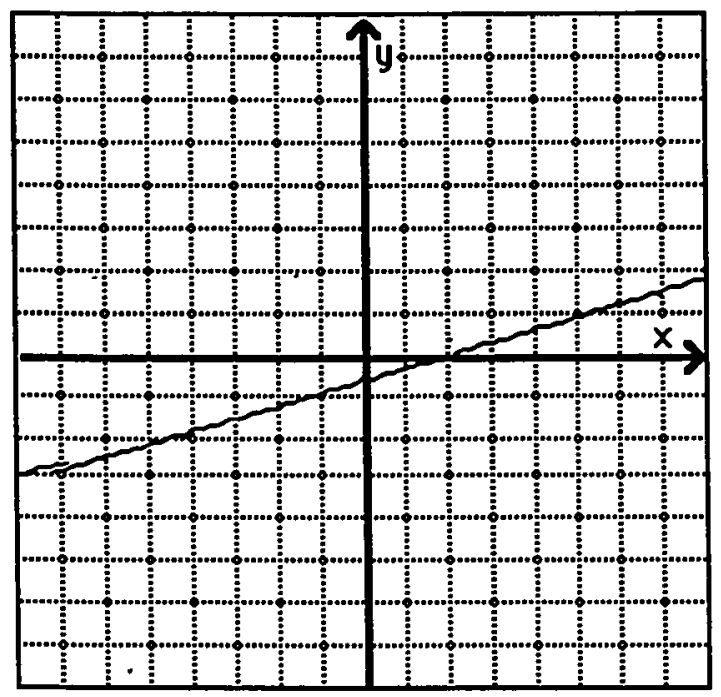

Part $d$ of question T3 was asked to see if the student could sketch the graph of the function just from the graph of the derivative function. The only acceptable answer was to graph an upright parabola with the vertex at approximately $x=2$.

For this question, only Tuong and Tylinn of the traditional group could sketch an acceptable graph for $g(x)$, and even Tuong had to calculate the equation of the line of $g^{\prime}(x)$ and take the antiderivative in order to sketch the function. The four incorrect responses are shown below. In the Harvard group, all six subjects were able to sketch an acceptable graph of $\mathbf{g}(\mathbf{x})$ (two sample sketches are seen below). None of the Harvard students used any algebraic techniques to aid their sketch. 
Tylinn's Sketch for Problem T3

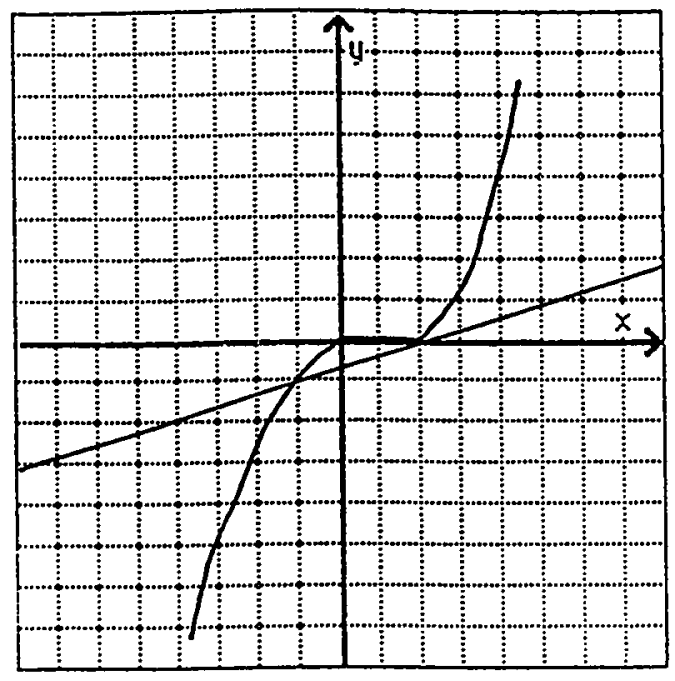

Tracy's Sketch for Problem T3

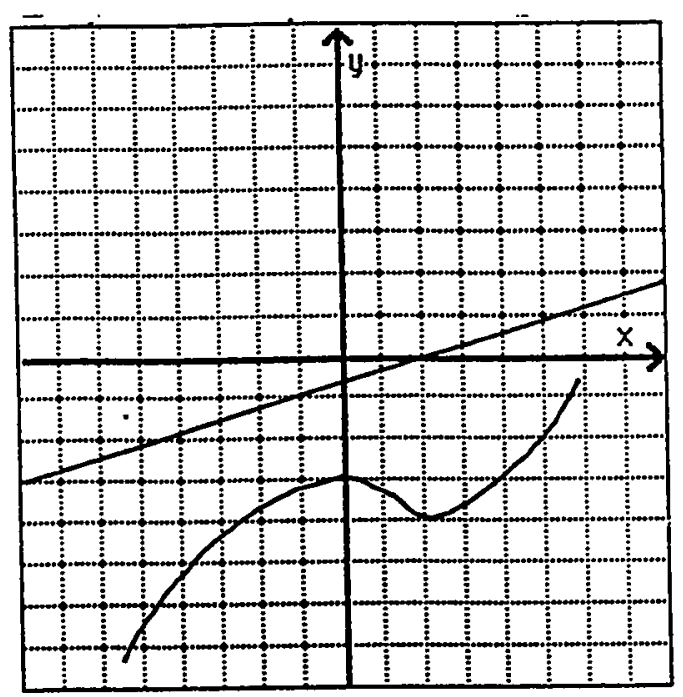

Tanya's Sketch for Problem T3

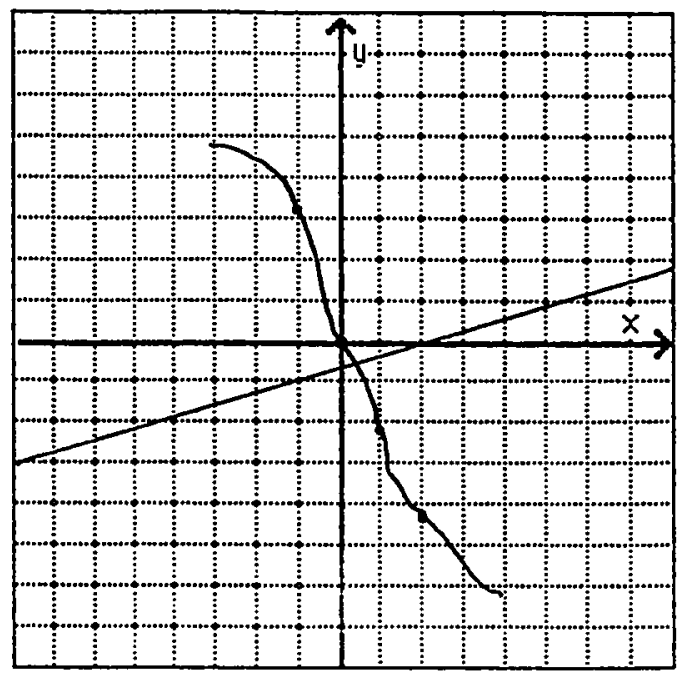

Tammy's Sketch for Problem T3

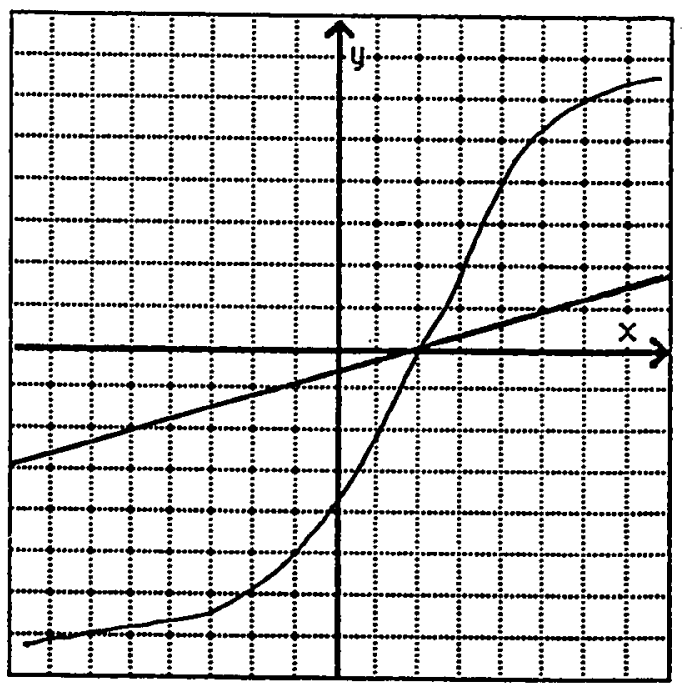


Henry's Sketch for Problem T3

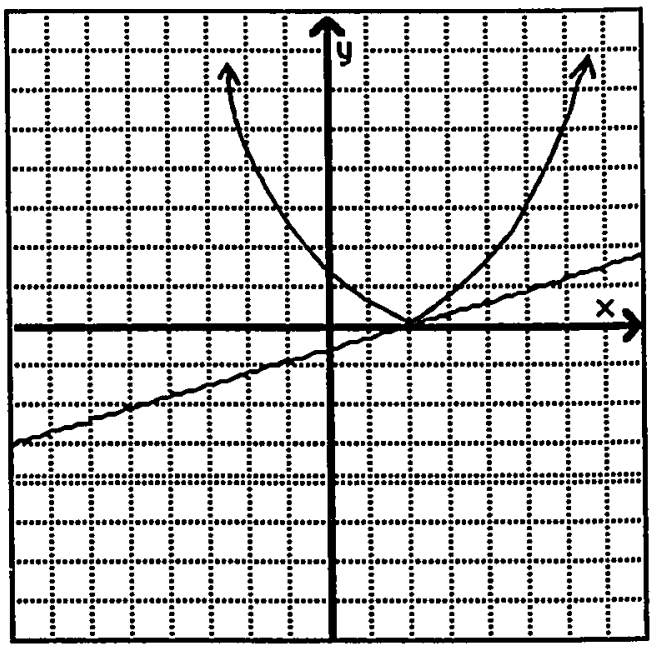

Hu-Anh's Sketch for Problem T3

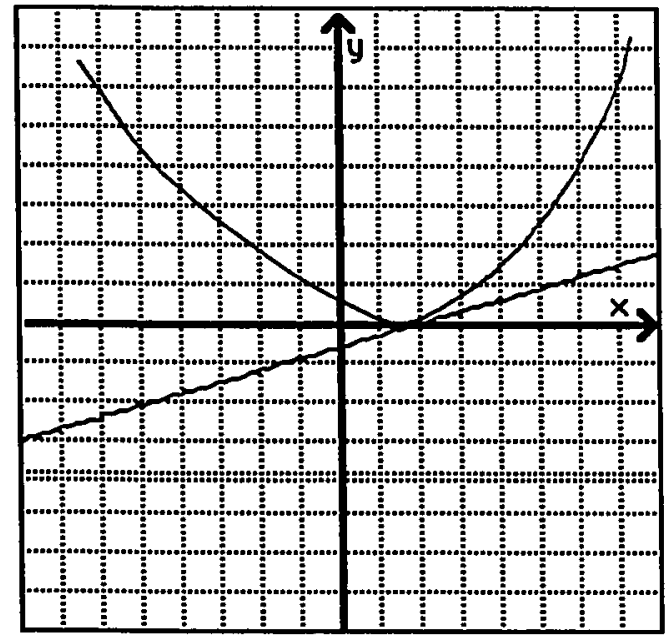

F14) By examining the graph, find $\int_{1}^{4} f(x) d x$.

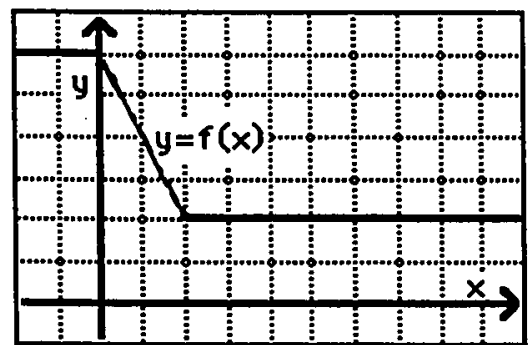

Five of the traditional students answered this correctly, although one of the five, Tuong, initially answered incorrectly because he tried to solve the problem algebraically. When asked if he could think of a quick way to check his work, he decided to add the area up and then found his error (he had used an incorrect equation initially). Only Tanya answered incorrectly. She tried to answer it algebraically also, only she made some errors. When asked if she could think of any other approach to the problem, she said no.

Five Harvard students also answered problem F14 correctly. They all found the area under the curve. Herb said he did not know how to solve the problem.

\section{Uniqueness}

Question F9 served as a followup question to question T3, part d. However, as you will see below, if their sketch for problem T3d was not correct, they were asked a 
similar question that probed the same idea.

F9) You have drawn a parabola and what I want to know is, is that the only function that would have that line for the graph of its derivative? In other words, is your solution unique?

Or, if they did not sketch an appropriate curve, ask the following: If the graph of the derivative of a function is a line, what would the graph of the original function look like? Would there be a unique solution?

These questions were asked to see if the subject understood that there is not a unique function for any given derivative. The only acceptable answer was to say that their parabola could be shifted vertically.

Only three of the five traditional students asked this question answered it correctly (as an oversight, Tanya was not asked this question). The two incorrect responses came from Tuong, who thought that the function was unique, and from Tylinn, who thought that only horizontal shifts of the function were acceptable.

Five of the Harvard students answered this correctly. Only Henry gave an unacceptable answer, thinking that the function was unique. Henry said that for that particular graph, "the derivative at the point $x=2$ is 0 , so the bottom of your curve where the tangent is 0 has to be sitting on $x=2$."

\section{Summany of Responses from Integration}

There were only three questions asked on integration, so any findings are not conclusive. The Harvard students did much better at the first two questions, but the groups performed equally on the third question. Table 6 summarizes their responses to sketching the function (given the graph of the derivative), solving the integral (graphically), and the uniqueness of the original function (given the derivative). 
TABLE 6: INTEGRATION

\begin{tabular}{lccc}
\hline Category/Question & Responses: & Good & Unacceptable \\
\hline Sketch & Traditional Group: & 2 & 4 \\
(Question T3d) & Harvard Group: & 6 & 0 \\
\hline Uniqueness & Traditional: & 3 & 2 \\
(Question F9) & Harvard: & 5 & 1 \\
\hline Solve & Traditional: & 5 & 1 \\
(Question F14) & Harvard: & 5 & 1 \\
\hline
\end{tabular}

Arranging the results topic-by-topic clearly illustrates the similarities and differences between the two groups of students. The groups performed similarly on functions, and there were students from both groups who thought continuity was a factor in determining functions. Also, although the traditional group did slightly better on limits, both groups did poorly on this important calculus topic.

The differences between the two groups can be seen by looking at the students' responses on continuity, differentiation, and integration. The Harvard group did noticeably better than the traditional group on those three topics. Also, the Harvard group seemed to work with the graphical representations better than the traditional group. These results will be carefully analysed and discussed in the next chapter. Also, the limitations of this study as well as some recommendations for future studies will be seen in Chapter 5. 


\section{CHAPTER 5}

\section{CONCLUSION}

The goal of this study was to compare the effectiveness of the traditionally taught calculus to Harvard Calculus. Specifically, this study was conducted to answer two questions: Whether or not the Harvard students are leaving class with 1) a better attitude about calculus and mathematics, and 2) a better understanding of the main calculus concepts. This was done by interviewing and testing twelve students who had completed a year of calculus, six from the traditional course and six from the Harvard course. In this chapter, we will discuss the conclusions that can be made by examining the results of the tests and interviews, the limitations and generalizability of this study, and possibilities for further research.

\section{CONCLUSIONS OF THE STUDY}

In this section, we will look at the differences between the two groups in their attitude towards: mathematics, the instruction they received in their calculus course, and their calculus textbooks. We will then analyze each group's understanding of function, limit, continuity, derivative, and integration, looking for both similarities and differences. How each group fared on the different representations used in the study (symbolic, graphic, and numeric) will also be noted, when appropriate, under each topic.

\section{STUDENT ATTITUDES}

After the initial interview, it was clear to this researcher that the Harvard group had a much better attitude about the nature of mathematics. They left their calculus course with the feeling that mathematics is not just "figur(ing) out some numbers" (Henry) or "follow(ing) formulas" (Harriet). They saw the importance in the theory behind the formulas, and realized, for the first time, that mathematics is not just a set of rules and procedures.

They also had positive feelings about how the course was taught, as revealed by their comments. For instance, Helen said in Harvard Calculus there were "a lot more descriptions of how you came to the conclusions that you came to instead of just a number 
answer." Hu-Anh said the instruction was "a lot different. In previous math [classes] all I had was 'here's a formula, plug in the numbers.' There was no real application, you don't see what the theory is behind it. In [Harvard] Calculus, I saw real applications." Harriet cited the use of the computer as being the primary difference in instruction, and felt that it helped illustrate some of the concepts.

The Harvard group also responded positively to the text. Judging by the students' comments, the Harvard text definitely addresses at least one of the MAA concerns -- that is, the text is organized to discourage "mimicry calculus." Hannah said that "every problem was different than the examples. Other texts are like you follow a pattern and that's it. But here you have to keep thinking differently for each one." Hu-Anh had a similar response, saying that "in other books, they give you an example and say 'here, do this.' In this book, if you understand, you don't need to see an example." Most of the Harvard students felt that the text was very readable, and Harriet even said that it reads "like an English book" as opposed to a typical mathematics text. The only student who did not like the text was Helen, who felt the book was "much more vague" than other mathematics texts and that the examples in the book did not give one enough information to complete the homework. Although Helen did not like the text, her response supports the other claims that the book is not simply a cookbook of examples.

In the traditional group, the only comments that students made about the course related to the level of difficulty. Three found it to be more difficult and three found it to be no more difficult than previous mathematics courses. Comments on the instruction were mainly on how fast the course went, such as Ted saying that the course "goes by a lot faster." Tuong added that in his class "they give you an exam like every two weeks." Other comments about the instruction include Tammy saying that the class "followed the book almost exactly" and that in calculus "you have to figure more things out on your own." There were no comments made even remotely similar to the comments made by the Harvard group.

The comments made on the text were basically about whether or not they liked the text. Tylinn thought the book was "too technical." Tanya just said the book was "pretty easy." Tammy said "I really like the book a lot. If I don't understand what's going on I just read the section and teach it to myself, and it's that easy." One could easily infer from Tammy's comment that what she meant was that she could look at the examples and figure out the homework problems. However, no followup was made on her statement, so her exact meaning is uncertain. Tracy said she "couldn't understand the text" and she "learned from the teacher, not the text." Tuong felt that previous mathematics texts he 
had used were better written and better organized than Stewart's Calculus (1991). "It took me a full semester to understand the way the book worked." Again, no comments similar to the Harvard group's comments were made by the traditional students, and there was nothing said that made one feel that the book was really helping the students learn the concepts.

\section{FUNCTIONS}

As one can see by reading the responses to the questions on functions, the groups performed almost identically. In fact, the only question that showed any differences between the groups was in recognizing functions; for that particular topic, the traditional group had three perfect scores compared to the Harvard group's one perfect score. However, overall the traditional group had five students with an acceptable or better response, while the Harvard group had four; therefore the difference was negligible.

Although there were no real differences among the groups, there are some findings worth reporting. First of all, it appears that familiarity and continuity, like the research has shown (see Dreyfus \& Eisenberg 1983; Ferrini-Mundy \& Graham 1994; Vinner 1987), played a role in identifying functions. Four of the twelve students, two from each group, said that continuity was necessary for a graph to represent a function (and let us not forget that these are $\mathrm{B}+$ or better students). One of the four, Herb, also said that he identified graphs as functions if the graph was familiar to him. If we were to generalize this, that tells us that fully one-third of our best students think continuity is necessary for a graph to represent a function. It is disturbing to think what this might imply for the average student.

Also, of the remaining eight students who had valid criteria for determining functions, half of them still misidentified at least one graph as a function, even though the graph directly violated their stated criteria. For instance, graph $G 7^{1}$ (the shaded region) was misidentified as a function by one student who said she used the vertical line test and another who said there should only be one output for any given input. Clearly G7 does not meet these criteria. It seems that students are willing to depart from their criteria if the graph is familiar, since every calculus student has seen graphs of regions.

1 Graph G7 was the most misidentified graph. A total of five students (three from the traditional group and two from the Harvard group) thought the shaded region represented a function. However, only two of these five had valid criteria for determining functions. 
Perhaps this is something that both curricula need to work on.

\section{LIMITS}

Neither group did that well on the topic of limits; however, by reviewing the traditional group's responses in this category, one can see that they performed slightly better than the Harvard group. In fact, one look at Table 3 (page 57) reveals the fact that the traditional group had higher scores in every category except the one on discontinuous functions for which the limit did not exist. There were only two questions on limits in which the Harvard group did better than the traditional group -- questions T1b and F11d.

Instead of just looking at the numbers of correct responses for each question, perhaps the best way to look at their responses is to analyze what each group understands about limits. With that in mind, the students' responses were analyzed to see if they understood three key facts about limits: that the left and right limits must be equal in order for the limit to exist; that the limit at a point can exist even if that point is not in the domain of the function; and that the value of the function at a point is not necessarily the value of the limit at that point. For the student to be given credit for understanding one of these three key topics, he/she must correctly answer each question that addresses that topic. If the student answered inconsistently, then he/she clearly was not sure and therefore was not given credit for understanding the topic.

After this analysis, it was clear that the traditional group had a better understanding of limits. In the traditional group, four of the students understood that the left and right limits must be equal in order for the limit to exist, while only two Harvard students understood this. Also, three of the six traditional students understood that a function need not be defined at a certain point in order for a limit to exist there; only two from the Harvard group knew that. Finally, three subjects from the traditional group knew that the value of the function at a point is not necessarily the same as the limit at that point, while only one from the Harvard group knew this. As stated in Chapter 2, Williams (1991) came to the conclusion that the current (traditional) curriculum does not seem to be teaching students the formal definition of limit; clearly the students taught with the Harvard curriculum have not learned it either.

Interestingly, one might have predicted early on that the Harvard group would do poorly on limits. In the initial interview, each subject was asked to name some of the key topics of calculus. As a whole, people from both groups mentioned functions, 
continuity, derivatives, and integration, and three people from the traditional group mentioned limits. However, not a single person from the Harvard group mentioned limits when asked to name the main topics of calculus. This may mean nothing, but together with the above results, it seems to tell us that the Harvard curriculum may need to spend more time on developing limits.

If one looks at the combined totals from both groups, one gets a fairly dismal view of what our students are learning about limits, regardless of the curriculum. Only six of the twelve students understood the relationship between the existence of the limit and the right and left sided limits. Less than half understood the other two key facts mentioned above. Again the reader is reminded that these are some of the best calculus students. If we expect calculus students to learn limits rigorously, perhaps some changes to both curricula will be necessary.

\section{CONTINUITY}

- The Harvard group did much better than the traditional group on the questions involving continuity. In fact, there was not a single question on continuity that the traditional group answered as well as the Harvard group.

In defining continuity, five of the Harvard students gave an acceptable or better definition. Only two students from the traditional group were able to do this. Also, it is interesting to note that two subjects from the traditional group defined continuity as the function being defined for every point in its domain; this view of continuity is identical to the one held by Sandy, the research subject of Ferrini-Mundy and Graham (1994) detailed in Chapter 2.

Recognizing continuous functions both symbolically and graphically was something that both groups did reasonably well on; the Harvard group answered the questions almost flawlessly while the traditional group made a few mistakes. The biggest surprise was that two students, both from the traditional group, could not identify continuous functions when presented graphically. This was originally thought to be an extremely easy question, and yet they both were very confused on what constitutes a continuous function. If this problem is widespread, perhaps the traditional curriculum should spend a little more time on continuous functions.

The claim that the traditionally taught calculus may need to spend more time on continuous functions is further supported by the following: only two students from the traditional group understood the relationship between differentiability and continuity; 
four from the Harvard group understood this relationship. Perhaps the Harvard group understood this better since the most obvious and intuitive way to approach this relationship is graphically. That is not to suggest that the traditionally taught calculus courses do not explore this topic using graphs, because they do. But since the Harvard students are exposed to more graphs on a regular basis, they may have an advantage understanding topics that are presented in this manner. Regardless of why, the fact remains that the Harvard group did better at understanding this topic.

\section{DIFFERENTIATION}

Differentiation is another topic in which the Harvard group did better than the traditional group. In fact, the traditional group struggled with many of the questions on differentiation. The Harvard group did better on every topic of differentiation, but the areas in which they showed a clear and substantial advantage were in estimating derivatives and in interpreting the graph of the derivative. Also, both groups did very poorly on recognizing differentiability.

There were three estimation problems, two of which could be solved by using the difference quotient or sketching a tangent line and estimating its slope (Q11 and F8a), while the third question must be answered either descriptively (verbally) or graphically (F3b). When looking at the students' responses to these questions, the first and most obvious conclusion is that the Harvard group is much better than the traditional group at estimating derivatives. If one looks at the total solution attempts for all three problems combined, one notices that the traditional group had only nine satisfactory solutions compared to the Harvard group's fifteen. Another important difference in the two groups' solution attempts is that for problems Q11 and F8a, the Harvard group used graphical means four times to estimate the derivative, while no one in the traditional group ever thought to sketch a tangent line. This shows a greater flexibility in representations, as well as a stronger intuitive connection between the derivative and the tangent line. Additionally, this suggests that the Harvard group has greater creativity when approaching problems, since neither Q11 or F8a were presented in a graphical manner.

The Harvard group also did very well interpreting the graph of the derivative, and as they did on the estimation problems, the traditional group did poorly. Recall that the students were given the graph of a derivative function, and from that graph the students were asked to relate three things about the original function: the minimum point, the 
maximum point, and the interval in which the original function is increasing. If we look at all of the solution attempts to the three parts of this question, we see that the traditional group had only eight acceptable responses while the Harvard group had fourteen. That means that the traditional group made more incorrect responses than correct responses. Perhaps most disappointing was the fact that only one-third of the traditional subjects knew where the minimum point was and the correct interval in which the function is increasing. One can only hope that this does not apply to all traditionally taught calculus students.

As stated above, neither group did very well recognizing differentiability. It was no surprise when the students struggled with the differentiability of piecewise functions presented symbolically. After all, as noted in Chapter 4, that required some fairly sophisticated calculus skills, in addition to understanding left-sided and right-sided derivatives. However, it was very surprising to see that only three of the twelve students recognized differentiability graphically. This seemed like such a trivial task, and yet three-fourths of the students could not answer it correctly. Surely recognizing differentiability is not the most important thing a calculus student learns, but one would hope that such a simple task would not be so easily forgotten.

In summary, the Harvard group showed a much better intuitive grasp of the derivative, as well as a stronger ability to think flexibly and creatively. They also related well to the graph of the derivative, and for the most part interpreted it correctly. Since calculus is a service course for many of the calculus students, estimation skills and the ability to think flexibly are vital. The traditional calculus curriculum evidently needs to address these issues.

\section{INTEGRATION}

Integration was the topic that was the least explored in the interviews and tests. In fact, there were only three questions that addressed integration (questions T3d, F9, and F14). Respectively, the three things the students were asked to do were 1) graph the original function given the graph of the derivative function, 2) state whether or not the graph of the function is unique, and 3) solve an integral when only given the graph of the function.

The Harvard group really showed its strength on the graphing problem. All six of the Harvard students were able to sketch an acceptable solution for question T3d, while only two traditional students were able to successfully complete this task. Also, recall 
that one of the two traditional students, Tuong, had to use algebraic skills along with symbolic integration before he could sketch the original function. Clearly Tuong had no real intuitive feel for the problem (further evidence of this is seen in his graph, which, due to his approach, has the minimum point slightly off from where it should be -- see below).

Tuong's Sketch for Problem T3

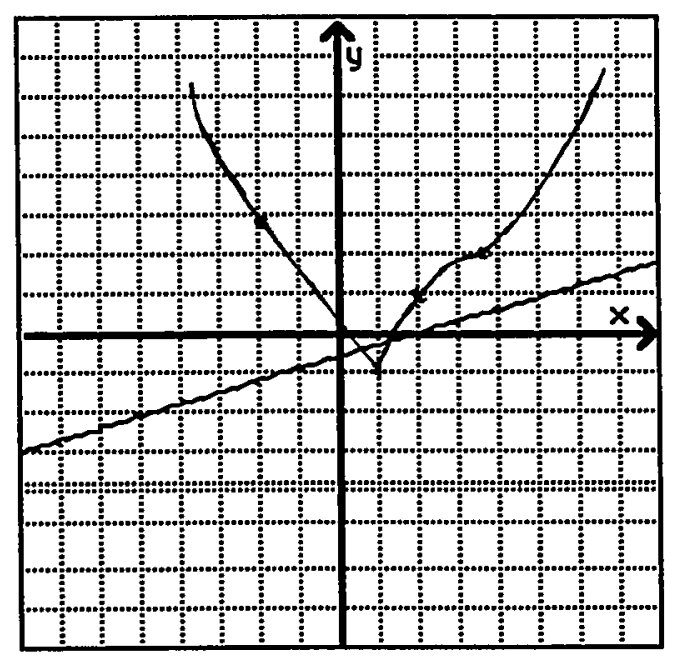

Perhaps the Harvard group's superior performance on this problem is due to the emphasis in the Harvard curriculum on graphical representations, but this definitely shows the Harvard group has a better intuitive grasp of integration.

Most of the students in the study understood that given the derivative function, the original function is unique up to vertical shifts. Eight out of the eleven students asked this question answered it correctly. Although five of the eight were from the Harvard group, Tanya was not asked this question; therefore, it is difficult to make any conclusions about the differences in the two groups. Also, even though only three out of eleven students missed this question, educators should not be too happy with that result; after all, the idea that the derivative function does not dictate one unique function is both basic and fundamental. One would think that all $A$ and $B$ students understood this.

The last question on integration proved to be very easy for almost everyone in the study. Five students from each group answered it correctly, although there was an interesting difference in the way the groups approached this problem. Four of the traditional students approached this problem graphically and two approached it alge- 
braically, even though this problem is much more difficult when approached from an algebraic point-of-view. All of the Harvard students except Herb approached this problem graphically; Herb did not know how to solve this integral. This is just more evidence that overall the Harvard group is more comfortable working in a graphical representation than the traditional group.

\section{SUMMARYOF CONCLUSIONS}

In this study, there were several conclusions made about the similarities and differences between the two groups. First, the Harvard group had a better attitude about mathematics and calculus, the instruction of their calculus classes, and the textbook. They indicated that the class was not simply a mimicry, or cookbook, course. Secondly, this study found that the two groups performed equally well on functions. The main problem encountered with functions was that some students from both groups thought a graph must be continuous for it to represent a function. The third finding from this study was that the traditional group had a better understanding of limits (although neither group truly excelled in this area). Both curricula should consider developing limits more rigorously. The fourth finding from this study was the Harvard group's overall dominance on the remaining topics (continuity, differentiation, and integration). The students from the Harvard group had a better understanding of continuity, differentiation, and integration. Finally, the Harvard group showed a greater flexibility in using various representations, and at times demonstrated much more creative thinking. The Harvard group did very well interpreting, sketching, and in general, working with graphs.

To summarize, there were four significant differences found between the two groups:

1) The Harvard group had a better attitude about mathematics.

2) The traditional group had a slightly better understanding of limits.

3) The Harvard group had a noticeably better understanding of continuity and differentiation, and did somewhat better on integration.

4) The Harvard group showed a greater flexibility in using various representations, and at times demonstrated much more creative thinking.

Clearly the Harvard group did better overall. They did as least as well on every topic 
except limits, and even with limits the differences were small.

Although these findings are important, one should not be tempted to overgeneralize the results. Like any study, this study has limitations. These limitations are discussed below.

\section{LIMITATIONS AND GENERALIZABILITY OF THE STUDY}

Although the interviews and tests generally went well, this study does have limitations and these limitations affect the generalizability of the conclusions. These limitations include the number and type $(B+$ or better) of students that were used, the timing of the study, and the questions asked and not asked.

First of all, there were only six students from each group. Although this was a qualitative and not quantitative study, it would have been better to have more students from each calculus series. This, unfortunately, could not be helped, because as stated in Chapter 3, there were exactly six students who had taken the Harvard calculus and who received a grade of $\mathrm{B}+$ or better, which leads us to the next potential problem. By using only the best students, the positive attitudes reported above are probably not indicative of all of the students in a Harvard calculus class. In fact, the professor of one of the two sections of Harvard calculus stated that several of her students complained about the course and the textbook, so these positive feelings are not universal.

Secondly, the timing of the study was not what it should have been, but again, this could not be helped. The study occurred almost four months after the end of the second semester of calculus. That means that the students had to recall some things from almost a year prior to the study. Retention may have played a factor in this study, although the timing was the same for both groups. Future studies should be conducted either while the students are still in their first or second semester of calculus, or immediately after (further recommendations for future studies are seen in the next section).

Finally, the questions asked (and not asked) played an important role in the results. For instance, perhaps a better selection of functions could have been used. Many of the limit questions as well as all of the continuity and differentiability questions used piecewise functions. Piecewise functions are often difficult for students to work with, a view that was voiced by Tanya. Others may have felt the same as Tanya without voicing their feelings. If that is the case, then some test errors may have been related to their understanding of piecewise functions and not the calculus concept in question. Also, there were a few questions that were not asked of some of the students. This happened 
because of 1) an oversight by the interviewer and 2) after the first two students took the followup test, a question was added. This played a very small role in the conclusions, but obviously it would have been ideal to have all subjects asked the exact same set of questions. There were also times when further followup questioning was needed but not conducted; this too may have played a small role in the conclusions, and was caused by the researcher's inexperience with the interview process.

\section{RECOMMENDATIONS FOR FURTHER RESEARCH}

As the number of reform calculus classes being offered continues to grow, more studies that compare the reform approach to the traditional approach should be conducted. Many of the questions from this study, in addition to some questions thought of or found in the research after this study was conducted, should be included in future studies.

The questions that were used in this study and that are recommended for future studies are the questions that seemed to reveal the most about students' understanding of the key concepts. That is, questions Q10 (recognize functions), Q11 (estimate derivative), T3 (interpret graph of derivative, sketch), F8 (estimate derivative), F11 (procedural limit questions), F12 (numerical/conceptual limit questions), and F15 (find the equation of the tangent line) are all recommended. Of course the other questions from this study should also be considered, but the above questions elicited interesting and telling responses.

Some additional questions were thought of that would be good to use in a comparative study like this one. For instance, in this study the students were shown the graph of the derivative function and asked to sketch an example of the original function. A good question to accompany that one is to show the students the graph of a function and ask them to sketch the derivative function. This idea was explored to some degree with question F3b, but the sketch of the original function was made by the student, so the researcher had little control over the function. Also, some questions on symbolic differentiation and integration might be useful to see if the traditional group has a clear advantage in this area.

For any future studies that want to explore students' understanding of limits, some excellent questions can be found in Williams' research (1991). For his study, he asked the participants to mark the following statements true or false: 
1) A limit describes how a function moves as $x$ moves toward a certain point.

2) A limit is a number or point past which a function cannot go.

3) A limit is a number that the $y$-values of a function can be made arbitrarily close to by restricting $x$-values.

4 ) A limit is a number or point the function gets close to but never reaches.

5 ) A limit is an approximation that can be made as accurate as you wish.

6 ) A limit is determined by plugging in numbers closer and closer to a given number until the limit is reached. (p. 221)

After marking the statements true or false, the students were asked to choose the one statement that in their opinion best described a limit. They were also to describe "what it means to say that the limit of $f$ as $x \rightarrow s$ is some number L." By asking these questions, along with some of the limit questions from the interviews and tests conducted for this paper, one should get a very good idea of what a student thinks about limits. This would be an excellent way of conducting a comparison test of understanding of limits.

In addition to using the questions mentioned above, on a practical note, future comparison studies could isolate one or two calculus topics and test exclusively on those: For example, one might compare a Harvard group and a traditional group on their views of differentiation, or perhaps study only limits and continuity. By isolating the topics, one can probe much deeper into the students' understanding of that particular topic, and have the freedom (and time) for greater followup. However, by isolating topics, one would lose some of the insights about the ways in which the topics are interconnected and related. Future researchers should be aware of this when choosing the content of their study.

\section{CLOSING STATEMENT}

Like Palmiter (1991) and Heid (1988) found, this report indicates that students' level of understanding of calculus concepts can be improved. By exploring real world problems using a variety of representations, Harvard Calculus has proven to be a worthy alternative to the traditionally taught calculus. If we want future calculus students to thoroughly understand the concepts, reform-style calculus may ultimately prove to be the only viable alternative. It is recommended that other Mathematics Departments offer a reform-style calculus, and perhaps follow up with a comparative study similar to this one. 


\section{REFERENCES}

Amit, M. \& S. Vinner. (1990). Some misconceptions in calculus: Anecdotes or the tip of an iceberg? Proceedings of the 14th International Conference for the Psychology of Mathematics Education, 1, (pp. 3-10). Oaxtepec, Mexico.: CINVESTAV.

Calculus Consortium at Harvard. (1994). Calculus. New York: John Wiley and Sons.

Cornu, B. (1991). Limits. In D.O. Tall (Ed.), Advanced Mathematical Thinking (pp. 153-166). Boston: Kluwer Academic Publishers.

Davis, R.B. \& S. Vinner. (1986). The notion of limit: Some seemingly unavoidable misconception stages. Journal of Mathematical Behaviour, 5, 281-303.

Douglas, R.G. (Ed.). (1986). Toward a lean and lively calculus. MAA Notes, No. 6. Washington, D.C.: Mathematical Association of America.

Dreyfus, T. \& T. Eisenberg. (1982). Intuitive functional concepts: A baseline study on intuitions. Journal for Research in Mathematics Education, 13, 360-380.

Eisenberg, T. (1993). Functions and associated learning difficulities. In D.O. Tall (Ed.) Advanced Mathematical Thinking (pp. 140 - 152). Boston: Kluwer Academic Publishers.

Ferrini-Mundy, J. \& K. Graham. (1991). An overview of the calculus curriculum reform effort: Issues for learning, teaching, and curriculum development. The American Mathematical Monthly, 98, 627-635.

Ferrini-Mundy, J. \& K. Graham. (1994). Research in calculus learning: understanding of limits, derivatives, and integrals. In J.J. Kaput and E. Dubinsky (Eds.) Research Issues in undergraduate mathematics learning: Preliminary analyses and results. MAA Notes, No. 33. Washington, DC: Mathematical Association of America.

Heid, M.K. (1988). Resequencing skills and concepts in applied calculus using the computer as a tool. Journal for Research in Mathematics Education, 19, No. 1, 325.

Kaput, J.J. \& E. Dubinsky (Eds.). (1994). Research Issues in undergraduate mathematics learning: Preliminary analyses and results. MAA Notes, No. 33. Washington, DC: Mathematical Association of America.

Monaghan, J. (1991). Problems with the language of limits. For the Learning of Mathematics, 11, 20-24. 
Norman, R. \& L. Prichard. (1994). Cognitive obstacles to the learning of calculus. In J.J. Kaput and E. Dubinsky (Eds.) Research Issues in undergraduate mathematics learning: Preliminary analyses and results. MAA Notes, No. 33. Washington, DC: Mathematical Association of America.

Orton, A. (1983a). Students' understanding of integration. Educational Studies in Mathematics, 14, 1-18.

Orton, A. (1983b). Students' understanding of differentiation. Educational Studies in Mathematics, 15, 235-250.

Palmiter, J.R. (1991). Effects of computer algebra systems on concept and skill acquisition in calculus. Journal for Research in Mathematics, 22, 151-156.

Scher, D. (1993). Students' conception of the derivative across multiple representations. Mathematics in College, Fall-Winter, 3-17.

Selden, J., A. Selden \& A. Mason. (1994). Even good calculus students can't solve nonroutine problems. In J.J. Kaput and E. Dubinsky (Eds.) Research Issues in undergraduate mathematics learning: Preliminary analyses and results. MAA Notes, No. 33. Washington, DC: Mathematical Association of America.

Sfard, A. (1992). Operational origins of mathematical objects and the quandary of reification -- the case of function. In G. Harel and E. Dubinsky (Eds.) The Concept of Function: Aspects of epistemology and pedagogy. MAA Notes and Reports, No. 25. Washington, DC: Mathematical Association of America.

Stewart, J. (1991). Calculus, 2nd Edition. Pacific Grove, CA.: Brooks/Cole Publishing Company.

Tall, D.O. (1980). The notion of infinite measuring numbers and its relevance in the intuition of infinity. Educational Studies in Mathematics, 11, 271-284.

Tall, D.O. \& R.L. Schwarzenberger. (1978). Conflicts in the learning of real numbers and limits. Mathematics Teaching, 82, 44-49.

Tall, D.O. \& S. Vinner. (1981). Concept image and concept definition in mathematics with particular reference to limits and continuity. Educational Studies in Mathematics, 12, 151-169.

Tucker, A.C. \& J.R.C. Leitzel (Eds.). (1995). Assessing calculus reform efforts. MAA Report, No. 6. Washington, D.C.: Mathematical Association of America.

Vinner, S. (1987). Continuous functions -- images and reasoning in college students. In J. Bergeron (Ed.), Proceedings of the 11th International Conference on the Psychology of Mathematics Education, Montreal: University of Montreal. 
Williams, S.R. (1991). Models of limits held by college students. Journal for Research in Mathematics Education, 22, 219-236.

Wilson, M.R. (1994). One preservice secondary teacher's understanding of function: The impact of a course integrating mathematical content and pedagogy. Journal for Research in Mathematics Education, 25, 346-370. 